\title{
Semiconductor Two-Dimensional PdQ2 (Q=S, Se) Monolayer: Strain Modulating Electronic Band Gaps and SQ Efficiencies
}

P Gajjar ( $\nabla$ pngajjar@gujaratuniversity.ac.in )

Gujarat University

Dhara Raval

Gujarat University

Sanjeev Gupta

St Xavier's College

Rajeev Ahuja

Uppsala University

\section{Research Article}

Keywords: 2D monolayer, Density functional theory, Band structure, Carrier mobility, SQ efficiency

Posted Date: December 3rd, 2021

DOI: https://doi.org/10.21203/rs.3.rs-1109301/v1

License: (c) (i) This work is licensed under a Creative Commons Attribution 4.0 International License.

Read Full License 


\title{
Semiconductor Two-dimensional $\mathrm{PdQ}_{2}(\mathrm{Q}=\mathrm{S}$, Se) monolayer: Strain Modulating
} electronic band gaps and SQ Efficiencies

\author{
Dhara Raval ${ }^{1}$, Sanjeev K. Gupta ${ }^{2,}$ *, P. N. Gajjar ${ }^{1, *}$ and Rajeev Ahuja $a^{3,4}$ \\ ${ }^{1}$ Department of Physics, University School of Sciences, Gujarat University, Ahmedabad \\ 380009, India. \\ ${ }^{2}$ Computational Materials and Nanoscience Group, Department of Physics and Electronics, \\ St. Xavier's College, Ahmedabad 380009, India. \\ ${ }^{3}$ Condensed Matter Theory Group, Department of Physics and Astronomy, Uppsala \\ University, Box 516, 75120 Uppsala, Sweden. \\ ${ }^{4}$ Department of Physics, Indian Institute of Technology Ropar, Rupnagar 140001, \\ Punjab, India.
}

Keyword (s): 2D monolayer, Density functional theory, Band structure, Carrier mobility, SQ efficiency

*Corresponding authors: Prof. (Dr.) P. N. Gajjar (E-mail: pngajjar@gujaratuniversity.ac.in) and Dr. Sanjeev K. Gupta (E-mail: sanjeev.gupta@sxca.edu.in) 


\begin{abstract}
We studied the physical, electronic transport and optical properties of a unique pentagonal $\mathrm{PdQ}_{2}(\mathrm{Q}=\mathrm{S}, \mathrm{Se})$ monolayers. The dynamic stability of $2 \mathrm{D}$ - wrinkle like - $\mathrm{PdQ}_{2}$ is proven by positive phonon frequencies in the phonon dispersion curve. The optimized structural parameters of wrinkled pentagonal $\mathrm{PdQ}_{2}$ are in good agreement with the available experimental results. The ultimate tensile strength (UTHS) was calculated and found that, penta-PdS $\mathrm{S}_{2}$ monolayer can withstand up to $16 \%(18 \%)$ strain along $x(y)$ direction with 3.44 $G P a(3.43 G P a)$. While, penta-PdSe $\mathrm{S}_{2}$ monolayer can withstand up to $17 \%(19 \%)$ strain along $x(y)$ dirrection with $3.46 \mathrm{GPa}(3.40 \mathrm{GPa})$. It is found that, the penta- $\mathrm{PdQ}_{2}$ monolayers has the semiconducting behavior with indirect band gap of 0.94 and $1.26 \mathrm{eV}$ for 2D-PdS 2 and 2D$\mathrm{PdSe}_{2}$, respectively. More interestingly, at room temperacture, the hole mobilty (electron mobility) obtained for $2 \mathrm{D}-\mathrm{PdS}_{2}$ and $\mathrm{PdSe}_{2}$ are 67.43 (258.06) $\mathrm{cm}^{2} \mathrm{~V}^{-1} \mathrm{~s}^{-1}$ and 1518.81 (442.49) $\mathrm{cm}^{2} V^{-1} \mathrm{~s}^{-1}$, respectively. In addition, $I-V$ characteristics of $\mathrm{PdSe}_{2}$ monolayer show strong negative differential conductance (NDC) region near the $3.57 \mathrm{~V}$. The ShocklyQueisser (SQ) effeciency prameters of $\mathrm{PdQ}_{2}$ monolayers are also explored and the highest $\mathrm{SQ}$ efficeinciy obtained for $\mathrm{PdS}_{2}$ is $33.93 \%$ at $-5 \%$ strain and for $\mathrm{PdSe}_{2}$ is $33.94 \%$ at $-2 \%$ strain. The penta- $\mathrm{PdQ}_{2}$ exhibits high optical absorption intensity in the $U V$ region, up to $4.04 \times 10^{5}$ (for $\mathrm{PdS}_{2}$ ) and $5.28 \times 10^{5}$ (for $\mathrm{PdSe}_{2}$ ), which is suitable for applications in optoelectronic devices. Thus, the ultrathin $\mathrm{PdQ}_{2}$ monolayers could be potential material for next-generation solar-cell applications and high performance nanodevices.
\end{abstract}




\section{Introduction}

Atomically thin two-dimensional (2D) structures like, honeycomb lattice graphene [1, 2], boron nitride (BN) [3, 4], transition metal dichalcogenides (TMDs), group - IV, V [5, 6] elements has recived attention due to their potential applications in the field of electronics, optoelectronics, solar cell, energy harvesting, and biomedical technologies with the high possibilities of replacing traditional 2D materials [1-7]. Among the 2D materials, if targeting application in nanodevices, $\mathrm{MX}_{2}(\mathrm{M}=\mathrm{Mo}, \mathrm{W}, \mathrm{V}, \mathrm{Ti}, \mathrm{Pd}, \mathrm{Pt} ; \mathrm{X}=\mathrm{S}, \mathrm{Se}, \mathrm{Te})$ type TMDs are technologically favourable materials [7-9]. Nowadays, an endless research is goingon traditional TMDs layered materials, which is in demand of high mobility, high stability in air and strain modulated bandgap. Of these, many of TMDs has been already synthesized via the various method of exfoliation [10-14]. Interestingly, palladium based dichalcogenides has brought 2D with buckled or puckered pentagonal structures under the spotlight because of its unique lattice arrangement and fascinating properties such as fine tuning their physical, elctronic, thermal and optical properties.

Gronvold et al., studied the $\mathrm{PdS} \mathrm{S}_{2} / \mathrm{PtS}_{2}$ and concluded that noble metals (Pd and $\mathrm{Pt}$ ) would be a layered structure with sulphur atoms [15, 16]. Afterwards, in 2015 Wang et al., theoretically reported that $2 \mathrm{D} \mathrm{PdS}_{2}$ is semiconductor with moderate indirect band gap up to $1.60 \mathrm{eV}$, which is very significant in fabrication of nanodevices [17]. Moreover, Cheng et al., [18] have developed ultrafast $\mathrm{Yb}$ doped fiber laser using few layers of $\mathrm{PdS}_{2}$ and indicated that layered $\mathrm{PdS}_{2}$ can be a favourable candidate for photonics application. Some work have also reported on the layered $\mathrm{PdSe}_{2}$ via method of mechanical exfoliated, atomic-resolution scanning transmission electron microscopy (AR-STEM) and chemical vapor deposition (CVD) [14, 19-24]. For Example, Akinola et al., [24] had fabricated few layers puckered pentagonal $\mathrm{PdSe}_{2}$ by AR-STEM method and demonstrated that, $\mathrm{PdSe}_{2}$ exhibits good ambipolar semiconducting nature with room temperature electron-apparent field-effect mobility (up to $\sim 158 \mathrm{~cm}^{2} V^{-1} \mathrm{~s}^{-1}$ ) and its stability is remain same up to 60 days. It has been noted that having high mobility (electron/hole) and band gap between $1.2 \sim 1.9 \mathrm{eV}$ of TMD, it makes them suitable for the FETs (field effect transistors) and CMOS applications, respectively [25-28].

Recently, Weiting and their research group [29] had successfully synthesized 2D $\mathrm{PdSe}_{2}$ nanosheets on $300 \mathrm{~nm} \mathrm{SiO} / 2 / \mathrm{Si}$ substract and claimed that it is really promising material to make infrared photodetector due to its high-photoresponsivity ( $660 \mathrm{~A}$. $\mathrm{W}^{-1}$ under $914 \mathrm{~nm}$ 
laser) in harsh condition, which may be benefitted in military field for night-time detection because of its ability to work at night as well as in bad whether conditions. Beside these, few other reports theoretical are also available on the $2 \mathrm{D}$ pentagonal type $\mathrm{PdQ}_{2}$ monolayers $[17$, 30-34]. Deng et al., [31] investigated the strain applied mechanical, electronic and optical properties of $\mathrm{PdS}_{2}, \mathrm{PdP}_{2}$ and $\mathrm{PtSe}_{2}$. Except this, none of works have been reported on strain applied mechanical, electronic, optical and electronic transport properties of $2 \mathrm{D}$ penta-PdQ 2 $(\mathrm{Q}=\mathrm{S}, \mathrm{Se})$. Further, the lower band gap of $2 \mathrm{D}-\mathrm{PdQ}_{2}$ monolayers has also motivated us to investigate absorbance of solar radiation on nanosheet. Hence, we also studied the strain dependent solar cell power conversion efficiency \% (PCE) of penta-PdQ $(\mathrm{Q}=\mathrm{S}, \mathrm{Se})$ monolayers.

In this work, we have investigated the geometric, mechanical, electronic transport and optical properties of penta-PdQ 2 using density functional theory (DFT). After the optimization of structures, we analysed the lattice parameters $(\AA)$, band gap $(\mathrm{eV})$ and effective mass $\left(m^{*}\right)$. Then after we applied biaxial strain $\varepsilon(\%)$ and obtained its bulk modulus $B(G P a)$. The ultimate tensile strength (UTHS) is also found under the tensile strain $(+\varepsilon \%)$ for $\mathrm{PdQ}_{2}$ $(\mathrm{Q}=\mathrm{S}, \mathrm{Se})$. We also studied the influence of loading on the band edges and obtained carrier mobility $\left(\mu_{2 D}\right)$ along biaxial strain $\varepsilon(\%)$. We have adopted Shockley-Queisser (SQ) method to study Solar cell efficiency $\eta(\%)$ and calculated the maximum power density $\left(P_{\max }\right)$. The optical properties including the dielectric constants, absorption co-efficient, refractive index, and reflectivity of penta- $\mathrm{PdQ}_{2}(\mathrm{Q}=\mathrm{S}, \mathrm{Se})$ monolayers are also reported in this paper. Herein, section 2 contains the methedology of computations, section 3 includes the results and disccusion part of the work on penta- $\mathrm{PdQ}_{2}(\mathrm{Q}=\mathrm{S}, \mathrm{Se})$ monolayers.

\section{Computational Methods}

The structural, electronic transport and optical properties of $2 \mathrm{D}$ wrinkled $\mathrm{PdQ}_{2}(\mathrm{Q}=\mathrm{S}, \mathrm{Se})$ monolayers were performed with in the SIESTA code [35]. The exchange-correlation functional approach was used in term of Perdew-Burke-Ernzerhof (PBE) type pseudopotential [36]. The basic unit cell of $2 \mathrm{D}-\mathrm{PdQ}_{2}(\mathrm{Q}=\mathrm{S}, \mathrm{Se})$ contains two $\mathrm{Pd}$ atoms and four $\mathrm{Q}(\mathrm{Q}=\mathrm{S}, \mathrm{Se})$ atoms as shown in Figure 1. An energy cut-off of 450 and $300 R y$ for $\mathrm{PdS}_{2}$ and $\mathrm{PdSe}_{2}$ monolayers were adopted, respectively. The doubled zeta plus (DZP) basis set was used with an energy of $0.02 R y$ to expand the Kohn-Sham orbital. The $\Gamma$ - centred mesh of $20 \times 20 \times 1$ and $15 \times 15 \times 1 \mathrm{k}$-points were sampled for $\mathrm{PdS}_{2}$ and $\mathrm{PdSe}_{2}$ monolayers, respectively under the Monkhorst-pack scheme in the 2D Brillouin zone [37]. The force $0.01 \mathrm{eV/A}$ was 
kept to relaxing the unstrained and strained structures of penta-PdQ $2(Q=S, S e)$ monolayers. The phonon dispersive curves were calculated using the density functional perturbation theory (DFPT) formalism [38]. In all calculation, the Fermi level $\left(E_{F}\right)$ is shifted at the zero energy. The parameters of carrier mobility of penta- $\mathrm{PdQ}_{2}(\mathrm{Q}=\mathrm{S}, \mathrm{Se})$ monolayers were found such as i.e., effective mass $\left(m^{*}\right)$, stiffness constants $\left(C_{2 D}\right)$ and deformation energy $\left(E_{1}\right)$ approximations using the following formula proposed by the Bardeen and Shockley [39], $\mu_{2 D}$ $=\frac{2 e \hbar^{3} C_{2 D}}{3 k_{B} T\left|m^{*}\right|^{2}\left(E_{1}\right)^{2}}$; here, $e$ is elementary charge of an electron, $\hbar$ is the reduced Planck's constant, $C_{2 D}$ is the in-plane stiffness constant and for $2 \mathrm{D}$ system it is defined as $C_{2 D}=$ $\frac{1}{S_{0}} \frac{\partial^{2} E}{\partial\left(\frac{a}{a_{0}}\right)^{2}}$; where $S_{0}$ is cell area and $a_{0}$ is the lattice constant at equilibrium, $E$ and $a$ are the total energy and lattice constant of the monolayer after deformation, $k_{B}$ is the Boltzmann constant, $T$ is the temperature $(300 \mathrm{~K})$. Also, $m^{*}$ is the effective mass in the transport direction and $\mathrm{E}_{1}$ is the deformation potential (DP) constant denoting the shifting of each band edges due to the applied biaxial strain $\varepsilon_{x y}$ defined as $E_{1}=\frac{\partial E_{e d g e}}{\partial\left(\frac{a}{a_{0}}\right)}$; where $E_{\text {edge }}$ is energy value of VBM (for holes) and CBM (for electrons).

The electronic transport properties of $\mathrm{PdQ}_{2}$ monolayers are calculated by utilizing the non-equilibrium Green's function (NEGF) in the form of $I-V$ response by TRANSIESTA module of SIESTA code [40, 41]. The electric current through the scattering region is calculated by exploring the Landauer-Buttiker formalism,

$$
I\left(V_{b}\right)=G_{0} \int_{\mu_{R}}^{\mu_{L}} T\left(E, V_{b}\right)\left[f\left(\mathrm{E}-\mu_{L}\right)-f\left(\mathrm{E}-\mu_{R}\right)\right] d E
$$

Where, $\mathrm{T}\left(\mathrm{E}, \mathrm{V}_{\mathrm{bias}}\right)$ is the transmission probability of an electron incident at an energy $\mathrm{E}$ through the device under the bias voltage $\mathrm{V}_{\text {bias }}, \mu_{L}$ and $\mu_{R}$ are the electrostatic potentials of left and right electrodes at a particular voltage bias and $\mathrm{G}_{0}$ is the unit of quantum conductance. The optical properties were investigated including the dielectric function and absorbance coefficient by DFT within the random phase approximation (RPA) [42]. The dielectric function is given by $\varepsilon(\omega)=\varepsilon^{\prime}(\omega)+i \varepsilon^{\prime \prime}(\omega)$. Here, $\varepsilon^{\prime}(\omega)$ and $\varepsilon^{\prime \prime}(\omega)$ are the real and imaginary parts of the complex dielectric function [43], respectively. 


\section{Results and Discussion}

\subsection{Stability and Mechanical Properties}

First, the geometric structures of pristine $2 \mathrm{D}$ penta- $\mathrm{PdQ}_{2}(\mathrm{Q}=\mathrm{S}, \mathrm{Se})$ were systemitically relaxed and lattice structures of penta- $\mathrm{PdQ}_{2}$ were obtained. It is built up with two $\mathrm{Pd}$ and four $\mathrm{Q}(\mathrm{S}, \mathrm{Se})$ atoms in the unitcell as shown in Figure 1(a, b). The differential charge density in Figure 1 (c, d) shows an accumulation and depletion of charges between Pd and Q (S, Se) atoms. In Table 1, the relaxed structural parameters are summarized with ground state properties such as, lattice parameter $(\AA)$, distance $\mathrm{d}(\AA)$ between Pd- $\mathrm{Q}_{1}, \mathrm{Pd}-\mathrm{Q}_{2}$ and $\mathrm{Q}_{1}$ $\mathrm{Q}_{2}$, thickness $t(\AA)$ and cohesive energy $E_{\text {coh }}(\mathrm{eV} / \mathrm{atom})$ of $\mathrm{PdQ}_{2}$ monolayers. 


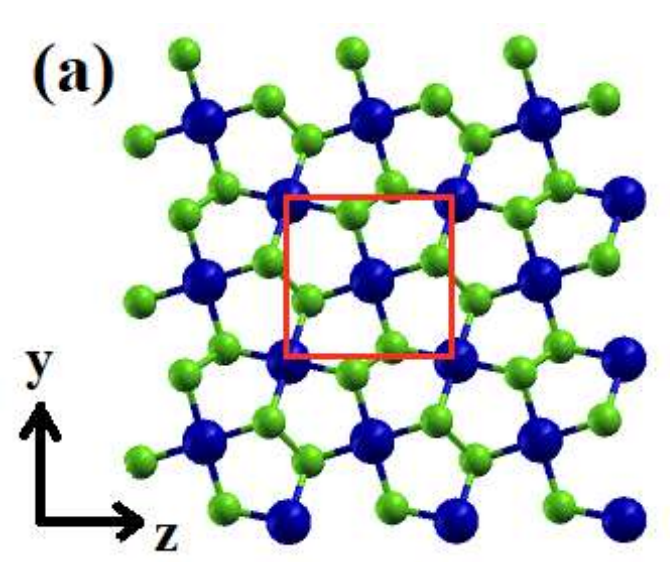

(c)

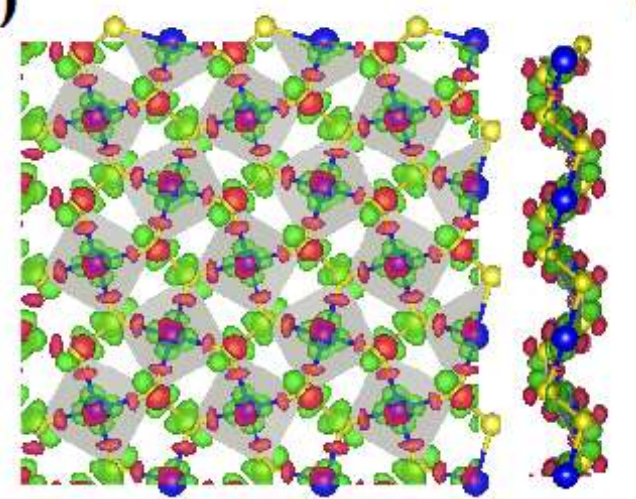

(b)
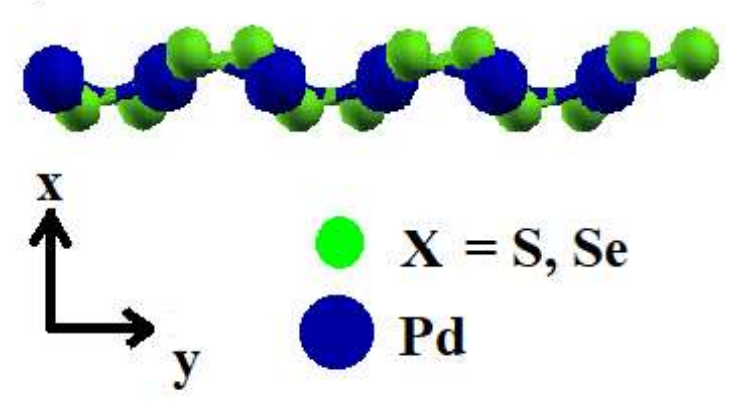

(d)

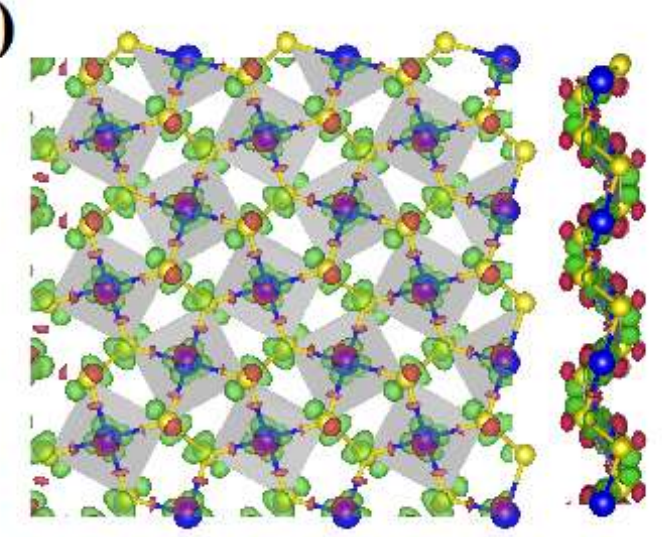

Figure 1. (Colour online) (a) Top-view of penta- $\mathrm{PdQ}_{2}(\mathrm{Q}=\mathrm{S}, \mathrm{Se})$ monolayer structure. Red line indicats the unitcell of the system. (b) Side views from $z$-direction of the atomic structure of penta-PdQ2. The navy blue and light-green circles represent the $\mathrm{Pd}$ and $\mathrm{Q}(\mathrm{S}, \mathrm{Se})$ atoms, respectively. The deformation charge density plot (c) for penta- $\mathrm{PdS}_{2}$ (d) for penta$\mathrm{PdSe}_{2}$; where red and green colour indicate the charge accumulation (electron excess) and charge deplation (electron loss). The iso-level is $0.063 e / \AA^{3}$ for both monolayers.

Table 1 : The calculated lattice parameter $(\AA)$, bond lengths $\mathrm{d}(\AA)$, thickness $\mathrm{t}(\AA)$ and cohesive energy $E_{c o h}(\mathrm{eV} / \mathrm{atom})$ of penta-PdQ 2 monolayers. 


\begin{tabular}{|c|c|c|c|c|c|c|c|}
\hline \multicolumn{2}{|c|}{$\begin{array}{c}\text { Penta-PdQ } 2 \\
(Q=S, S e)\end{array}$} & \multirow{2}{*}{$\begin{array}{l}\mathbf{d}_{\text {Pd-Q1 }}^{(\AA)} \\
2.35\end{array}$} & \multirow{2}{*}{$\begin{array}{c}\mathbf{d}_{\mathbf{P d}-\mathbf{Q} 2} \\
(\AA) \\
2.34\end{array}$} & \multirow{2}{*}{$\begin{array}{c}\underset{\mathbf{d} \times 1-\mathbf{Q} 2}{(A)} \\
2.14\end{array}$} & \multirow{2}{*}{$\begin{array}{c}\text { Thickness } \\
\left(\begin{array}{c}(\AA) \\
\end{array}\right) \\
1.29\end{array}$} & \multirow{2}{*}{$\begin{array}{c}\text { Lattice parameter } \\
\qquad(\stackrel{\AA}{A}) \\
a=5.62, b=5.42\end{array}$} & \multirow{2}{*}{$\begin{array}{c}\begin{array}{c}\text { Cohesive } \\
\text { energy } \\
\text { Ecoh } \\
\text { (eV/atom) }\end{array} \\
-4.71\end{array}$} \\
\hline Penta- & $\begin{array}{l}\text { Present } \\
\text { work }\end{array}$ & & & & & & \\
\hline $\mathbf{P d S}_{2}$ & $\begin{array}{l}\text { Other } \\
\text { DFT/ } \\
\text { *Exp. }\end{array}$ & $\begin{array}{l}2.34[17] \\
2.35[32]\end{array}$ & $\begin{array}{l}2.35[17] \\
2.34[32]\end{array}$ & $\begin{array}{l}2.10[17] \\
2.11[32]\end{array}$ & 1.28 [32] & $\begin{array}{l}a=5.49, b=5.59[17] \\
a=5.48, b=5.59[32] \\
a=5.48, b=5.57[33]\end{array}$ & -- \\
\hline \multirow[b]{2}{*}{$\begin{array}{l}\text { Penta- } \\
\text { PdSe }_{2}\end{array}$} & $\begin{array}{l}\text { present } \\
\text { work }\end{array}$ & 2.45 & 2.45 & 2.42 & 1.47 & $a=5.94, b=5.68$ & -4.30 \\
\hline & $\begin{array}{l}\text { Other } \\
\text { DFT/ } \\
\text { *Exp. }\end{array}$ & $2.45[34]$ & 2.46 [34] & $2.42[34]$ & -- & $\begin{array}{c}a=5.72, b=5.80[29] * \\
a=5.74, b=5.91[32] \\
a=5.75, b=5.92[33] \\
a=5.71, b=5.90[34]\end{array}$ & -- \\
\hline
\end{tabular}

As shown, in Table 1, the obtained lattice parameters $a(b)$ of $\mathrm{PdS}_{2}$ and $\mathrm{PdSe}_{2}$ monolayers are 5.62 (5.42) $\AA$ and 5.94 (5.68) $\AA$, respectively. The atomic distance between $\mathrm{Pd}-\mathrm{S}_{1}\left(\mathrm{Se}_{1}\right)$, $\mathrm{Pd}-\mathrm{S}_{2}\left(\mathrm{Se}_{2}\right)$, and $\mathrm{S}_{1}\left(\mathrm{Se}_{1}\right)-\mathrm{S}_{2}\left(\mathrm{Se}_{2}\right)$ atoms are 2.35 (2.45) $\AA, 2.34$ (2.42) $\AA$ and 2.14 (2.42) $\AA$, respectively and shows good agreement with previously reported results [17, 29, 32-34]. To evaluate stability of resulted structures, the cohesive energy is calculated using the formula: $E_{c o h}=\sum n_{X} E_{X}-E_{P d Q 2} / N$. where $E_{P d Q 2}$ denotes total energy of $\mathrm{PdQ}_{2}(\mathrm{Q}=\mathrm{S}, \mathrm{Se})$ unit cell, $N$ is total atom in unit cell and $n_{X}$ and $E_{X}$ are number of atoms of each element and atomic energies of each atom in the unit cell, respectively. It is found that cohesive energies for $\mathrm{PdS}_{2}$ and $\mathrm{PdSe}_{2}$ monolayer are -4.70 and -4.30 eV/atom, respectively. Although this is larger than that for the 2D-antimony (-4.03), 2D-arsenene (-2.96), 2D-MoS 2 (-4.11), 2D-MoSe 2 (-3.94) and 2D-black phosphorous (-3.48). This is evidence that penta- $\mathrm{PdQ}_{2}$ monolayers have strong bonding in its ring-network. In addition, to confirm the kinetic stability of penta- $\mathrm{PdQ}_{2}(\mathrm{Q}=\mathrm{S}$, Se) structures, we calculated the phonon dispersion curves along high symmetric points for both $\mathrm{PdS}_{2}$ and $\mathrm{PdSe}_{2}$ monolayers and results are as shown in Figure $\mathbf{S 1}(\mathbf{a}, \mathbf{b}), \mathbf{E S I}^{\dagger}$. The highest phonon frequencies extended is up to $2275 \mathrm{~cm}^{-1}$ and $2193 \mathrm{~cm}^{-1}$ for $\mathrm{PdS}_{2}$ and $\mathrm{PdSe} \mathrm{C}_{2}$ monolayers, respectively. Evidently, no negative acoustics branch is appeared in the first Brillouin zone, itself proves the dynamical stability of both the monolayers at room temperature.

Next, we focused on mechanical properties of penta-PdQ 2 and evaluated Young modulus $Y(N / m)$ and bulk modulus $B(N / m)$. The Young moduli $Y(N / m)$ and bulk moduli $B$ 
$(\mathrm{N} / \mathrm{m})$ were investigated under uniaxial and biaxial strain on the penta-PdQ 2 monolayers. The range of applied strain $\varepsilon(\%)$ is taken in interval of $-5 \% \leq \varepsilon \leq+5 \%$ in the step of $1 \%$. The bulk modulus computed by applying biaxial load ( $x$ and $y$ ) on the penta-PdQ 2 monolayer with following relation, $[44,45]$

$$
B=\mathrm{S}_{0} \frac{\partial^{2} E_{S}}{\partial A^{2}}
$$

Where, $S_{0}$ is unstrained cross-sectional area of the unit cell. $E_{s}$ and $A$ are the total strain energy and area of respected applied strain, respectively. Figure $\mathbf{S 2}(\mathbf{a}, \mathbf{b}), \mathbf{E S I}^{\dagger}$ shows the strain energy vs area curve for pristine and loaded structures of $\mathrm{PdS}_{2}$ and $\mathrm{PdSe}_{2}$ monolayers, respectively. This curve is fitted by the polynomial curve fitting and with help of Eq. (1), we have obtained bulk modulus $B(N / m)$ as shown in Figure S2 $(\mathbf{a}, \mathbf{b})$, ESI ${ }^{\dagger}$. The calculated bulk modulus $(\mathrm{N} / \mathrm{m})$ of $\mathrm{PdS}_{2}$ and $\mathrm{PdSe} \mathrm{S}_{2}$ are 30.22 and $23.56 \mathrm{~N} / \mathrm{m}$, respectively. These values are larger than that for the monolayer of $\mathrm{Sb}(\sim 21.88 \mathrm{~N} / \mathrm{m})$ and As $(\sim 25.78 \mathrm{~N} / \mathrm{m})[44,46]$, that indicate that penta-PdQ 2 has stiffer and better resistance to deformation compared to antimony ( $\mathrm{Sb}$ ) and arsenene (As) monolayers. Afterwards, we have calculated the in-plane Young modulus $Y(N / m)$ in longitudinal (along $x$-axis) or transverse (along $y$-axis) direction for penta-PdQ2. To obtained Young modulus from first-principles calculation, the following formula was employed [44, 45],

$$
Y=\frac{1}{S_{0}} \frac{\partial^{2} E_{S}}{\partial \varepsilon^{2}}
$$

Here, $S_{0}$ is unstrained cross-sectional area of the unicell. Next, $\frac{\partial^{2} E_{S}}{\partial \varepsilon^{2}}$ shows the second derivative of strain energy $(e V)$ with applied load. Here, the compressive and tensile strains are applied to the penta- $\mathrm{PdQ}_{2}$ in the longitudinal or transverse direction. The Young moduli obtained by using the curves shown in Figure S3 $(\mathbf{a}, \mathbf{b}), \mathbf{E S I} \mathbf{I}^{\dagger}$. The calculated Young modulus $\mathrm{Y}(\mathrm{N} / \mathrm{m})$ along $x(y)$ direction are $74.13(42.40) \mathrm{N} / \mathrm{m}$ and $65.26(28.62) \mathrm{N} / \mathrm{m}$ for penta-PdS 2 and penta-PdSe 2 , respectively. The results indicating that, Young modulus decreases as we switched $\mathrm{S}$ to Se, and this is due to the increment in the Pd-S and Pd-Se bond strength. Xiong et al. [47] had calculated orientation-dependent Young modulus of $2 \mathrm{D}$ orthorhombic $\mathrm{MX}_{2}$ $(\mathrm{M}=\mathrm{Ni}, \mathrm{Pd} ; \mathrm{X}=\mathrm{S}, \mathrm{Se}, \mathrm{Te})$ and reported values are $\sim 50$ (37) $\mathrm{N} / \mathrm{m}$ for $\mathrm{PdS}_{2}\left(\mathrm{PdSe}_{2}\right)$. From the present investigation of Young modulus of penta- $\mathrm{PdQ}_{2}$, we suggest that the $2 \mathrm{D}$ penta-PdQ 2 can be preferred candidates for flexible devices because of having ultra-low values of Young modulus, compared to that of $2 \mathrm{D}$ graphene $(340 \mathrm{~N} / \mathrm{m})$ and $2 \mathrm{D} \mathrm{MoS}_{2}(125 \mathrm{~N} / \mathrm{m})[48,49]$. 

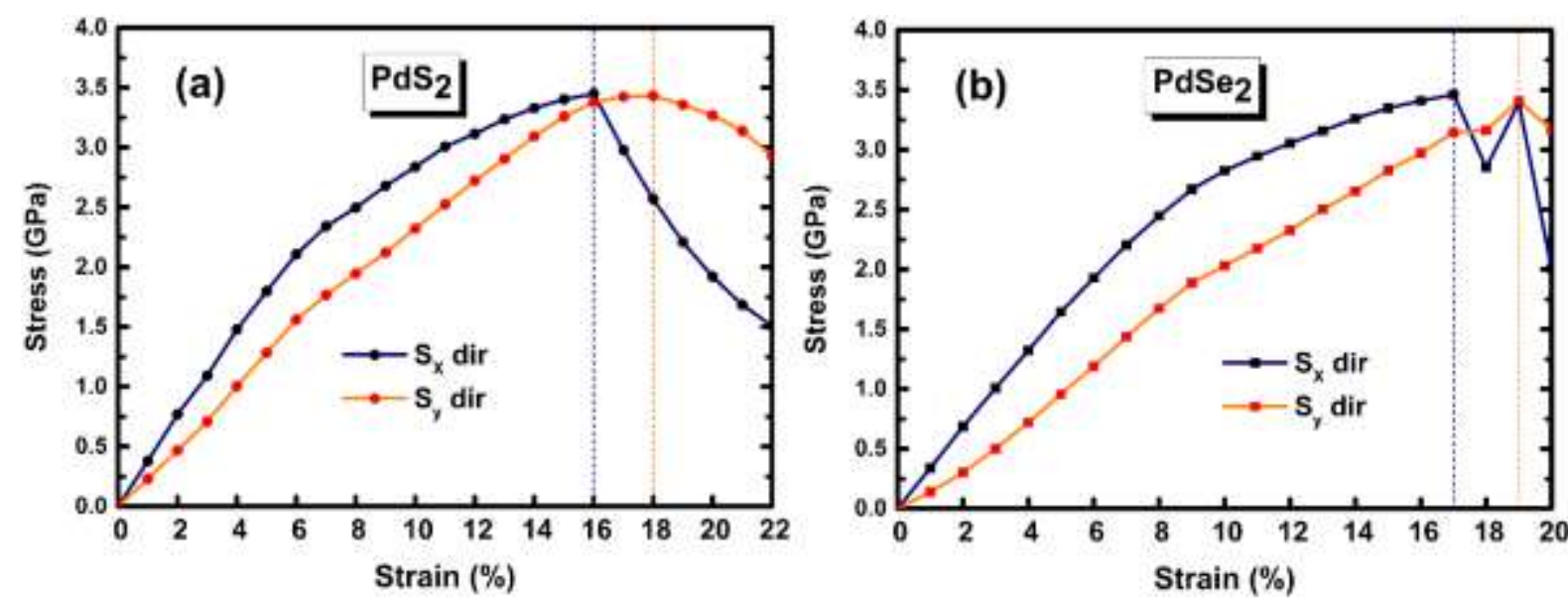

Figure 2. (Colour online) The stress (GPa) vs strain (\%) curve under tensile strain. (a) For penta-PdS 2 and (b) For penta- $\mathrm{PdSe}_{2}$.

Further, we have also explored the mechanical stability of penta-PdQ 2 monolayers and examined that up to which values of strain (\%) the monolayers can withstand? It is worth to find curve of stress-strain relation called ultimate tensile strength (UTSH) curve [50]. The UTSH is representing the maximum stress value $(G P a)$ that a monolayer can withstand prior to the fractured structure set. This can be calculated by the components of stress tensor with respect applied strain. The computed UTSH for penta-PdQ $\mathrm{P}_{2}$ monolayers are shown in Figure 2 (a, b). The stress value, where the slope of the stress-strain curve becomes zero indicates the value of UTSH, and the strain at this value represents the magnitude of ultimate tensile strain (UTSR). In case of penta-PdS 2 , the calculated values of UTSR along $x(y)$ directions is 16\% (18\%) at stress of $3.44 \mathrm{GPa}$ and $3.43 \mathrm{GPa}$, respectively (see Figure 2(a)). Whereas, in penta-PdSe $\mathrm{Pse}_{2}$ Figure 2(b)) it is observed at 17\% (19\%) with stress value of $3.46 \mathrm{GPa}$ (3.40) $G P a$. The present values of UTSR for penta-PdQ 2 are higher than the bluephosphorene monolayer ( 16\%) [51]. Thus, the UTSR values suggest that the penta-PdQ 2 monolayer is relatively more flexible.

\subsection{Electronic Properties}

The band structure, total density of states (TDOS) and partial density of states (PDOS) of penta- $\mathrm{PdQ}_{2}$ monolayers along high symmetry points of Brillouin zone (BZ) have also investigated. At PBE level, from Figure 3 (a, b) we can see that the penta- $\mathrm{PdS}_{2}\left(\mathrm{PdSe}_{2}\right)$ is an indirect band gap with valence band maximum (VBM) and conduction band minimum (CBM) tracing at S-point (M-point) and $\Gamma$-point, respectively. The obtained indirect band 
gap of penta-PdS $\mathrm{P}_{2}$ is $\mathrm{E}_{\mathrm{g}}{ }^{\mathrm{PBE}}=0.94 \mathrm{eV}$, while band gap for penta-PdSe${ }_{2}$ is $\mathrm{E}_{\mathrm{g}}{ }^{\mathrm{PBE}}=1.26 \mathrm{eV}$ as seen in Figure 3 (a, b). We also did calculations using GGA+U, LDA, LDA+U and HSE06 approximations for $\mathrm{PdS}_{2}\left(\mathrm{PdSe}_{2}\right)$ and obtained indirect band gaps are 1.05 (1.26) eV, 0.92 (1.31) $\mathrm{eV}, 0.94$ (1.30) and $0.92 \mathrm{eV}$, respectively. Next, having heavier element (Pd) in 2D penta-PdQ 2 material, the spin-orbit coupling (SOC) effect may play a crucial role and could influence in the band gap $\left(E_{g}\right)$ of material. Thus, we have also checked the SOC effect on the penta-PdQ 2 monolayer and results are shown in Figure $\mathbf{S 4}(\mathbf{a}, \mathbf{b}), \mathbf{E S I}^{\dagger}$. Using $\mathrm{PBE}+\mathrm{SOC}$ functional calculation, the obtained indirect band gap of penta- $\mathrm{PdS}_{2}$ is $\mathrm{E}_{\mathrm{g}}{ }^{\mathrm{PBE}+\mathrm{SOC}}=1.16 \mathrm{eV}$, whereas band gap of penta-PdSe ${ }_{2}$ is $\mathrm{E}_{\mathrm{g}}{ }^{\mathrm{PBE}+\mathrm{SOC}}=1.51 \mathrm{eV}$. Recently, the Yang et al [32], have reported electronic properties of penta-PdS $\mathrm{S}_{2}$ from $\mathrm{PBE}(\mathrm{PBE}+\mathrm{SOC})$ calculation and reported indirect band gap of $1.08 \mathrm{eV}(1.13 \mathrm{eV})$, which is comparable with our results within of $12.96 \%(11.5 \%)$ deviation. Moreover, a research group of Sun et al [34], have reported a band gap of penta-PdSe $\mathrm{S}_{2}$ with PBE level theory and mentioned indirect band gap of $1.38 \mathrm{eV}$, which is higher by $8.6 \%$ than our obtained band gap $(\sim 1.26 \mathrm{eV})$. Furthermore, with SOC effect, Qin et al [33], has computed indirect band gap of penta-PdSe $\mathrm{S}_{2}$ monolayer via TB-mBJGGA potential and reported band gap $1.38 \mathrm{eV}$. The deviation of present band gap of penta$\mathrm{PdSe}_{2}$ with $\mathrm{PBE}+\mathrm{SOC}$ calculation is $9 \%$. The presently obtained values are close to optimal value of $1.3 \mathrm{eV}$ for solar cell applications. As presented in Figure $3(\mathbf{a}, \mathbf{b})$, it is noticed that the TDOS (States/eV) are mostly ascend due to ' $d$ ' orbitals of Pd atom and ' $p$ ' orbitals of $\mathrm{Q}$ $(\mathrm{S}, \mathrm{Se})$ atom in the penta-PdQ 2 monolayer, which are also consistent to the past reported results $[32,34]$. Additionally, the TDOS difference from PBE to PBE+SOC calculations are shown in Figure S5 (a, b), (ESI $\left.{ }^{\dagger}\right)$ for $\mathrm{PdS}_{2}$ and $\mathrm{PdSe}_{2}$ monolayers. we have also investigated PDOS (States/eV) as displayed in Figure $3(\mathbf{c}, \mathbf{d})$, to further look into partial contribution of atoms in penta-PdQ 2 and we conclude that the conduction bands $(\mathrm{CBs})$ and valence bands (VBs) are highly occupied by the $4 d-P d$ states and $3 p-Q(\mathrm{Q}=\mathrm{S}, \mathrm{Se})$ states, respectively. 

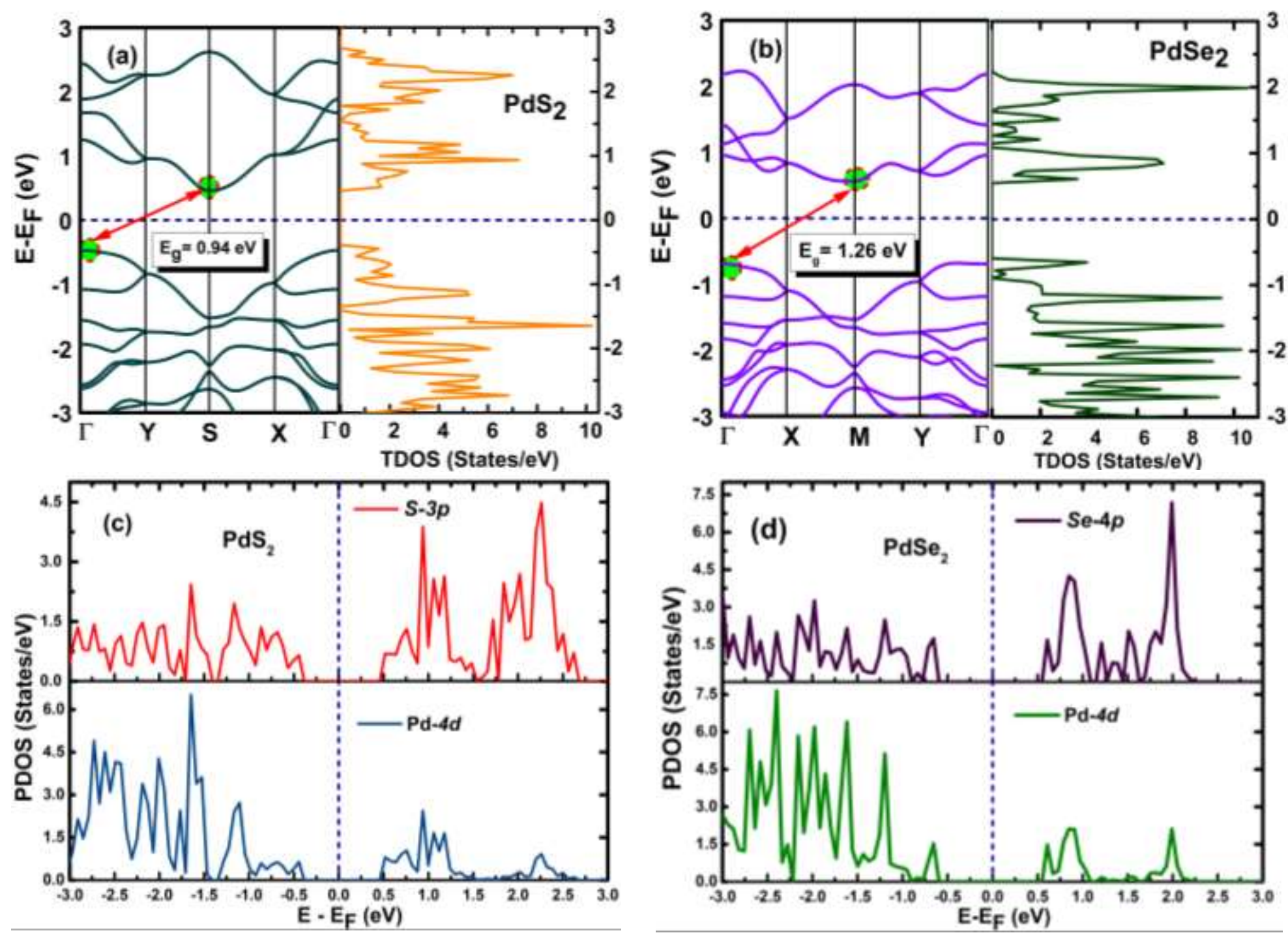

Figure 3. (Colour online) Electronic band structure and total density of states (TDOS) States/eV (a) For penta-PdS $\mathrm{S}_{2}$ monolayer and (b) For penta-PdSe $\mathrm{S}_{2}$ monolayer. The partial density of states (PDOS) States/eV (c) For penta-PdS $\mathrm{S}_{2}$ monolayer and (d) For penta-PdSe 2 monolayer.

\subsection{Transport Properties}

\subsubsection{Carrier Mobility}

We further focused on the band alignment in terms of strain effect to drive the electronic properties. Simultaneously, we have also calculated carrier mobility $(\mu)$ of monolayer, which is one of essential factor for a high-performance device like FET. For this, we have exerted compressive $(-\varepsilon \%)$ and tensile $(+\varepsilon \%)$ strain on penta- $\mathrm{PdQ}_{2}(\mathrm{Q}=\mathrm{S}, \mathrm{Se})$ monolayers and traced 
influence on the band edges of CBM and VBM in the band structure, where system is anisotropic ( $a$ not equal to $b$ ). The strength of strain along biaxial direction is given by [52], $\varepsilon$ $=\left[\left(S-S_{0}\right) / S_{0}\right] \times 100 \%$; here, $S_{0}$ is the unstrained lattice constant and $S$ is the strained lattice constant of the monolayer. The response of applied strain to band gaps of penta$\mathrm{PdQ}_{2}$ is shown in Figure 4.

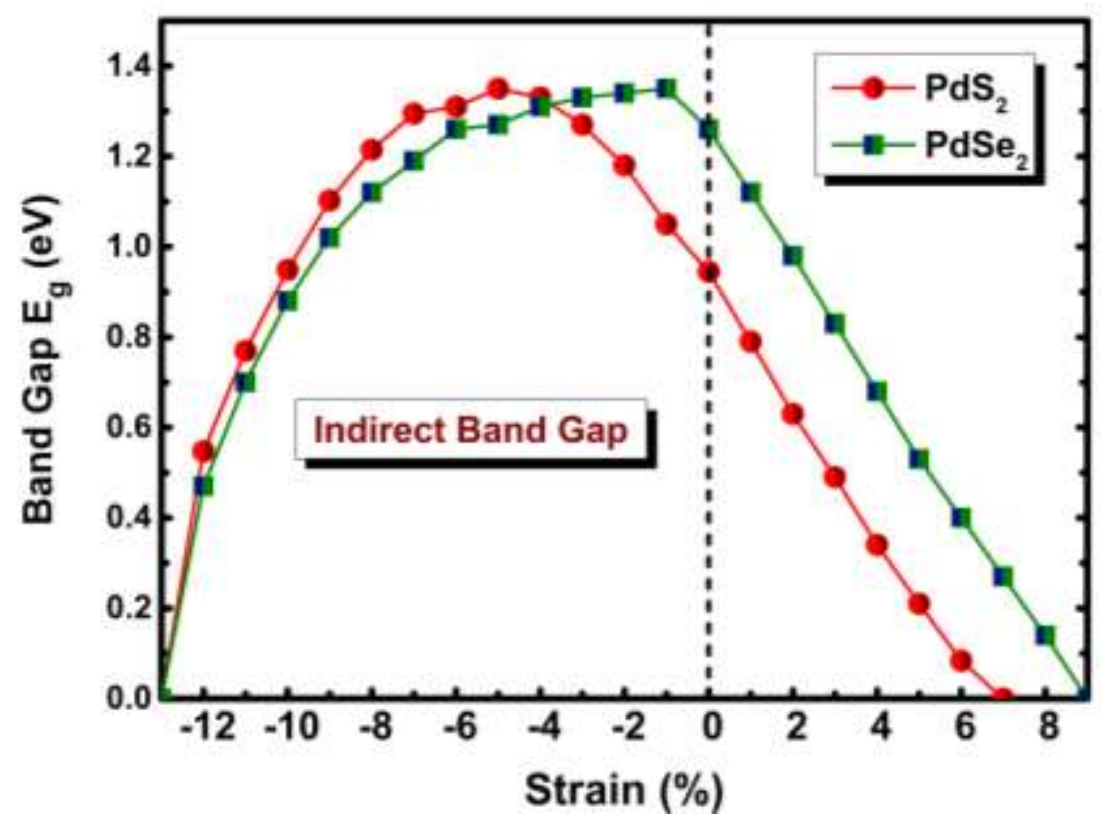

Figure 4. (Colour online) The variation of band gap $\mathrm{E}_{\mathrm{g}}(\mathrm{eV})$ with applied biaxial strain $\varepsilon(\%)$ on penta-PdQ 2 monolayers.

As stated before, the fundamental indirect band gap of penta- $\mathrm{PdS}_{2}$ and penta-PdSe 2 is $0.96 \mathrm{eV}$ and $1.26 \mathrm{eV}$, respectively. The Figure 4 reflects that the band gap of penta- $\mathrm{PdQ}_{2}$ is gradually decreases almost linearly in presence of tensile strain $(+\varepsilon \%)$ compared to fundamental band gap (0\%). Interestingly, upon increases compressive strain (- $\varepsilon \%)$ band gap of penta-PdS $\mathrm{S}_{2}$ increases up to $-5 \%$ compressive strain and then decreases gradually with respect to $0 \%$. It is noteworthy that, the maximum value of band gap of penta-PdS $\mathrm{S}_{2}$ is $\sim 1.35$ $e V$ at $\varepsilon=-5 \%$ and for penta- $\mathrm{PdSe}_{2}$ is $\sim 1.36 \mathrm{eV}$ at $\varepsilon=-1 \%$. Remarkably, the resistive phase transition is seen at higher compressive $(-\varepsilon \%)$ and tensile strain $(+\varepsilon \%)$ in both cases. The semiconducting nature of penta-PdS $\mathrm{S}_{2}\left(\mathrm{PdSe}_{2}\right)$ is transform into semi-metallic $(\sim 0 \mathrm{eV})$ by applying the biaxial compressive and tensile strain of $-13 \%(-13 \%)$ and $+7 \%(+9 \%)$, respectively. Under the critical compressive strain $(-\varepsilon \%)$, the resistive phase change (semiconductor to semi-metallic) in penta- $\mathrm{PdQ}_{2}$ behaviour arises due to crossing one band of VBM at the Fermi level (Figure S6 (a, b), ESI $\left.{ }^{\dagger}\right)$. In contrary, up on the critical tensile $(+\varepsilon \%)$ 
strain, it occurred due to touching one band of CBM at the Fermi level (Figure S7 (a, b), $\left.\mathbf{E S I}^{\dagger}\right)$. Additionally, we have also examined the phonon spectrum for each applied strain on penta-PdQ 2 and computed its phonon frequency as summarized in Table S1, ESI ${ }^{\dagger}$. Thus, our outcomes accomplish those electronic properties of studied $2 \mathrm{D}$ penta- $\mathrm{PdQ}_{2}(\mathrm{Q}=\mathrm{S}, \mathrm{Se})$ materials are tuneable by applying biaxial strain, which establish that the penta-PdQ 2 is a promising candidate for designing flexible nanodevices.

Table 2. The carrier effective mass $\left|m^{*}\right|\left(\mathrm{m}_{\mathrm{e}}\right.$, the mass of free electrons), deformation

\begin{tabular}{|c|c|c|c|c|c|c|}
\hline System & $\begin{array}{c}\text { Carrier } \\
\text { type }\end{array}$ & $m * / m_{e}$ & $E_{1}(e V)$ & $\begin{array}{c}C_{2 D} \\
\left(J m^{-2}\right)\end{array}$ & $\left(\mathrm{cm}^{2} \stackrel{\mu}{v^{-1} s^{-1}}\right)$ & $R=\mu_{h} / \mu_{e}$ \\
\hline \multirow[t]{2}{*}{ Penta-PdS2 } & electron & 0.39 & -6.44 & 121.76 & 258.06 & \multirow{2}{*}{0.26} \\
\hline & hole & 0.72 & 6.85 & 121.76 & 67.43 & \\
\hline \multirow[t]{2}{*}{ Penta-PdSe2 } & electron & 0.58 & -2.91 & 94.62 & 442.49 & \multirow[t]{2}{*}{3.43} \\
\hline & hole & 0.14 & 6.14 & 94.62 & 1518 & \\
\hline
\end{tabular}

potential constant $E_{1}(\mathrm{eV})$, stiffness constant $C_{2 D}\left(\mathrm{~J} \mathrm{~m}^{-2}\right)$, carrier mobility ( $\mu$ ) for electron (e) and hole $(\mathrm{h})$ and carrier mobility ratio $(R)$ along biaxial direction of penta- $\mathrm{PdQ}_{2}$ monolayers at $300 \mathrm{~K}$.

Owing to tunability of penta- $\mathrm{PdQ}_{2}$ monolayers, we inspired to calculate the carrier mobility of penta-PdQ 2 monolayers. For that the Bardeen and Shockley formalism [39] had been applied, we have calculated the effective mass $\left(\mathrm{m}^{*}\right)$, deformation potential $E_{1}(\mathrm{eV})$ and stiffness constant $C_{2 D}\left(\mathrm{~J} \mathrm{~m}^{-2}\right)$ to check the flow of charge carrier along biaxial strain. The carrier mobility depends on the most influenced factor on the carrier effective mass $m^{*}$ of electron (e) and hole (h), that is directly derived from the electronic band structure as [53],

$$
m^{*}=\hbar^{2}\left\{\frac{d^{2} E}{{d k^{2}}^{2}}\right\}^{-1}
$$

Next, deformation potential $E_{l}$ is another vital parameter to effect carrier mobility and is obtained from the deformation theory (DP) [39], which has been also successfully used method in previous reports for $2 \mathrm{D} \mathrm{MoS}, \mathrm{WS}_{2}$ and phosphorene. The needed ingredients for carrier mobility $(\mu)$ are summarized in Table 2. Along biaxial strain, the obtained effective mass $\left(m^{*}\right)$ of electron and hole for penta-PdS $2\left(\mathrm{PdSe}_{2}\right)$ are $0.39 m_{e}\left(0.58 \mathrm{~m}_{\mathrm{e}}\right)$ and $0.72 m_{e}$ 
$\left(0.14 m_{e}\right)$, respectively. Our computed deformation potential $E_{1}(\mathrm{eV})$ for penta- $\mathrm{PdS}_{2}$ is -6.44 $e V$ for e and $6.84 \mathrm{eV}$ for h, that is consistent with ones reported by Wang et al [17], along $x$ or $y$ direction. While in case of penta-PdSe 2 , the value of $E_{1}$ is $-2.91 \mathrm{eV}$ for $e$ and $6.14 \mathrm{eV}$ for $h$ that is also in accordance with the results of Qin et al, [33] for $x$ or $y$ direction. Whereas the in-plane stiffness constant $C_{2 D}$ for penta- $\mathrm{PdQ}_{2}$ has anisotropic behaviour. The in-plane stiffness constant obtained for penta-PdS 2 and penta-PdSe 2 are $121.76 \mathrm{~J} \mathrm{~m}^{-2}$ and $94.62 \mathrm{~J} \mathrm{~m}^{-2}$, respectively. The stiffness constant $C_{2 D}$ of penta- $\mathrm{PdS}_{2}$ is larger than the penta-PdSe indicating that $\mathrm{PdSe}_{2}$ monolayer is softer than the penta-PdS $\mathrm{S}_{2}$ monolayer. As shown in Table 2, the achieved electron mobility of penta-PdS 2 monolayer is $258 \mathrm{~cm}^{2} \mathrm{~V}^{-1} \mathrm{~s}^{-1}$, which is quite higher than the hole mobility $\sim 67 \mathrm{~cm}^{2} V^{-1} \mathrm{~s}^{-1}$. Also, the carrier mobility ratio R comes out to be 0.26. Moreover, Wang et al [17] has reported the electron mobilities $\left(\mathrm{cm}^{2} V^{-1} \mathrm{~s}^{-1}\right)$ of penta$\mathrm{PdS}_{2}$ as $40.97(x)$ and $169.11(y)$, while hole mobilities $\left(\mathrm{cm}^{2} V^{-1} \mathrm{~s}^{-1}\right)$ as $339.25(x)$ and 91.73 $(y)$. On other hand, if we compare carrier transport along biaxial strain of penta-PdSe 2 the hole mobility is $1518 \mathrm{~cm}^{2} \mathrm{~V}^{-1} \mathrm{~s}^{-1}$ that is greater than the electron mobility $442.49 \mathrm{~cm}^{2} \mathrm{~V}^{-1} \mathrm{~s}^{-1}$ and $\mathrm{R}$ is 3.43 . However, it is observed that the hole mobility of penta-PdSe 2 is higher than the 2D phosphorene [54] (i.e., 640-700 $\mathrm{cm}^{2} \mathrm{~V}^{-1} \mathrm{~s}^{-1}$ ) and $\mathrm{BN}$ nanosheet (i.e., $500 \mathrm{~cm}^{2} \mathrm{~V}^{-1} \mathrm{~s}^{-1}$ ) [55], indicating that penta-PdSe 2 would be a promising material for modelling electronic applications.

\subsubsection{Current-Voltage (I-V) Characteristics}

To understand the current sensitivity of the penta-PdQ 2 monolayers, we have examined the I-V characteristics, based on the equivalent transport theory [41]. Figure 5 represents the schematic view of two-terminal device, where we have considered LE (left electrode), RE (right electrode) and scattering region of same material.

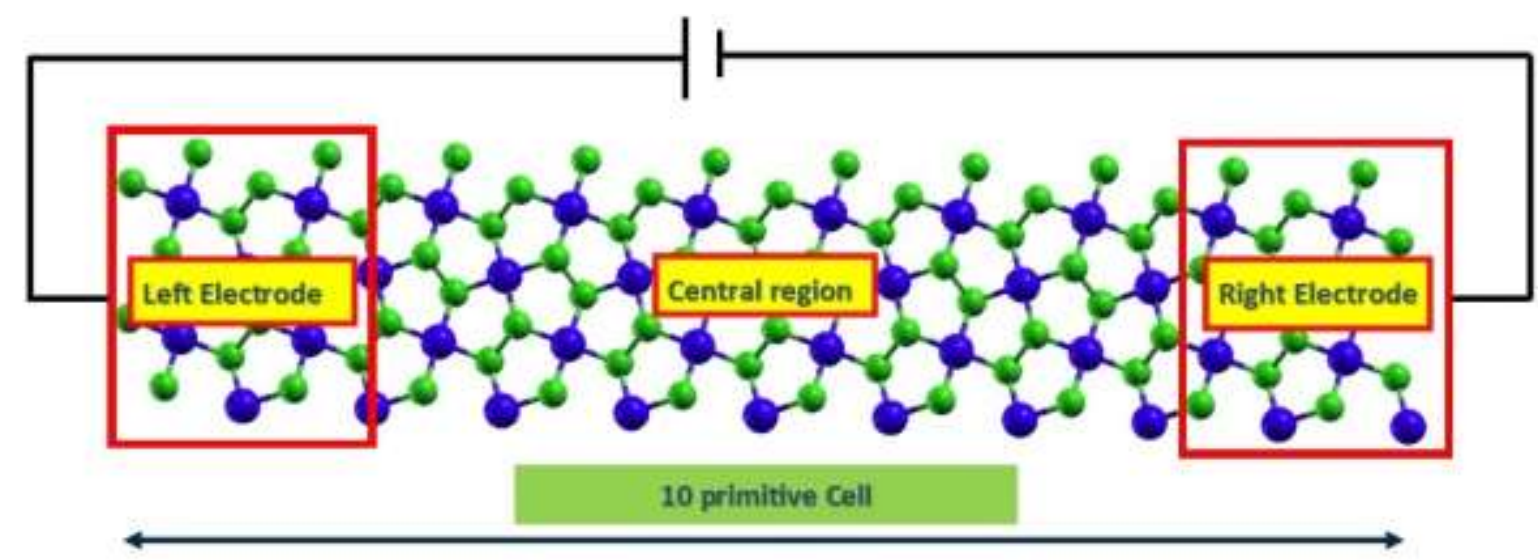


Figure 5. (Colour online) Schematic view of two-terminal device of penta-PdQ 2 monolayer. Here electrons are driven from the cold to the hot lead through central region.

Here, we have taken 10 primitive cells, among them the lead had built up with fourunit cell (24 atoms) and central region consist of 12-unit cells (72 atoms). The I-V characteristic of both penta-PdS 2 and penta-PdSe $\mathrm{P}_{2}$ monolayers are shown in Figure $6(\mathbf{a}, \mathbf{b})$. It is seen that, no significant current is observed till $\sim 1.0 \mathrm{~V}$ and $1.5 \mathrm{~V}$ then it starts to increase with increasing applied voltages due to semiconducting nature of penta-PdS $\mathrm{S}_{2}$ and $\mathrm{PdSe}_{2}$ monolayers, respectively. Apparently, it can be seen form Figure $6(\mathbf{a}, \mathbf{b})$ penta- $\mathrm{PdS}_{2}$ is more current-sensitive than the penta-PdSe 2 monolayer. Although, in the case of penta-PdS $\mathrm{S}_{2}$, the first peak in current is seen at $2.5 \mathrm{~V}$ with $14 \mu \mathrm{A}$ and second current peak is located at $4.0 \mathrm{~V}$ with current of $13.2 \mu \mathrm{A}$. This is indicating a pronounced negative differential conductance effect (NDC) [56] in the bias range 2.0-3.5 V (see Figure 6(a)). While in case of penta-PdSe, the NDC effect is seen in range of 1.0-4.5 $V$ (see Figure 6(b)). More precisely, the first NDC effect is occurs at the bias voltage $1.0 \mathrm{~V}$ and current to be found about $0.678 \mu \mathrm{A}$. Further, the second and third peaks are occurring at the bias voltage of $3.0 \mathrm{~V}$ and $4.5 \mathrm{~V}$ with current up to $1.35 \mu A$ and $2.05 \mu A$, respectively. The NDC effect is very useful feature in $I-V$ curve because it would be playing a crucial role in the application of multipliers, mixers, logic gates, high-frequency oscillators, and A to D (analog-to-digital) converters [57].
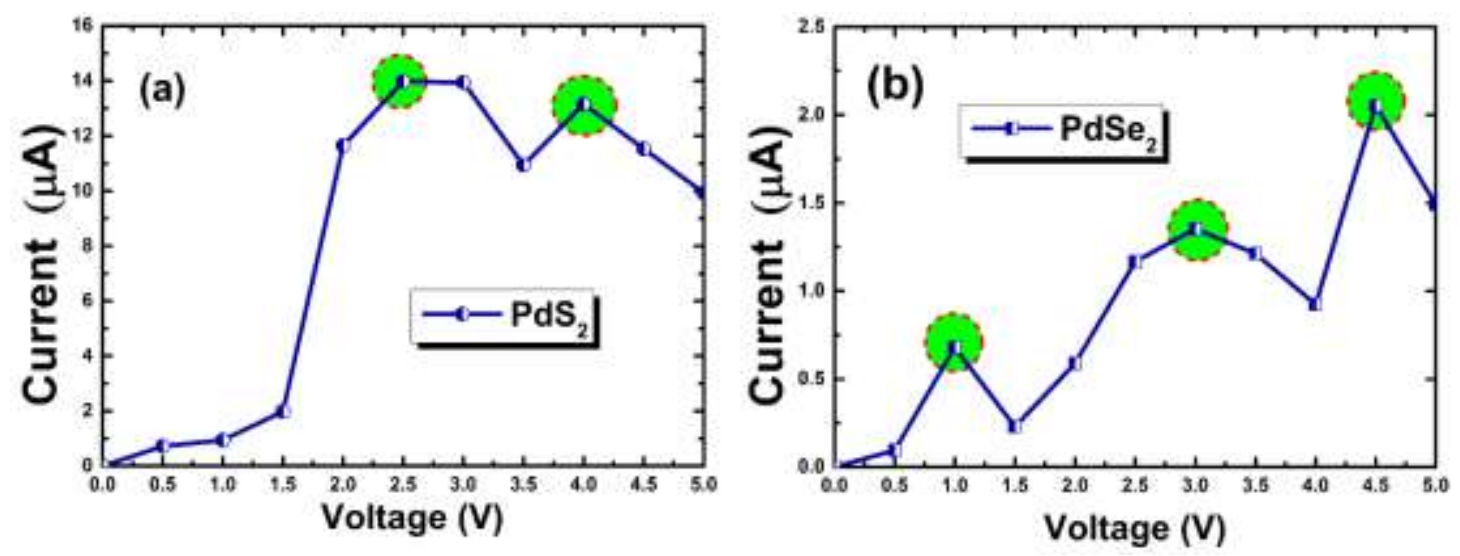

Figure 6. (Colour online) Current-voltage $(I-V)$ plot of penta-PdQ 2 under bias voltages from $0.0 \mathrm{~V}$ to $5.0 \mathrm{~V}$. The negative differential conductance (NDC) regions are indicated by the highlighted circles.

\subsection{SQ Efficiency}


We also focused on the power conversion efficiency (PCE) of penta-PdQ $2(Q=S, S e)$ using Shokley-Queisser (SQ) limit that gives the percentage of power converted to electrical energy $[58,59]$. For this we required several parameters such as short circuit current density $\left(J_{s c}\right)$, open circuit voltage $\left(V_{o c}\right)$, maximum power density $\left(P_{\max }\right)$ and efficiency $\eta(\%)$. The theoretical short circuit current density $J_{s c}$ is calculated using following relation $[60,61]$,

$$
J_{s c}=\int_{0}^{\infty} e A(E) I_{s u n}(E) d E
$$

where $e$ is the elementary charge, $A(E)$ represents the absorbance of layer [60] and $I_{\text {sun }}(E)$ is the photon flux density as specified in the AM1.5G spectrum [62], Also, the theoretical reverse saturation current $J_{0}$ is defined as $J_{0}=\int_{0}^{\infty} e \pi A(E) I_{b b}(E) d E[63]$; Here $I_{b b}(E, T)$ is the black body spectrum and the radiative recombination fraction, which is supposed to be unity [58]. Thus, the total current density can be obtained using the formula [63],

$$
J=J_{s c}-J_{0}\left(\exp \left(\frac{e . V}{k . T}\right)-1\right)
$$

where $k$ is the Boltzmann's constant, $V$ is the voltage over the absorber of the $2 \mathrm{D}$ devices. By the relation of $P=J V$, the maximum power density $P_{\max }$ can be evaluated from the maxima of the $J-V$ curve, as presented in Figure 7(a, b). Eventually, with help of above-mentioned parameters, now we have obtained solar cell efficiency $\eta(\%)$ by formula [59],

$$
\eta=\frac{P_{\max }}{P_{\text {in }}}
$$

where $P_{\text {in }}\left(1000 \mathrm{~W} / \mathrm{m}^{2}\right)$ is total incident power density from solar irradiation of AM1.5G and $P_{\max }$ is maximum power density.
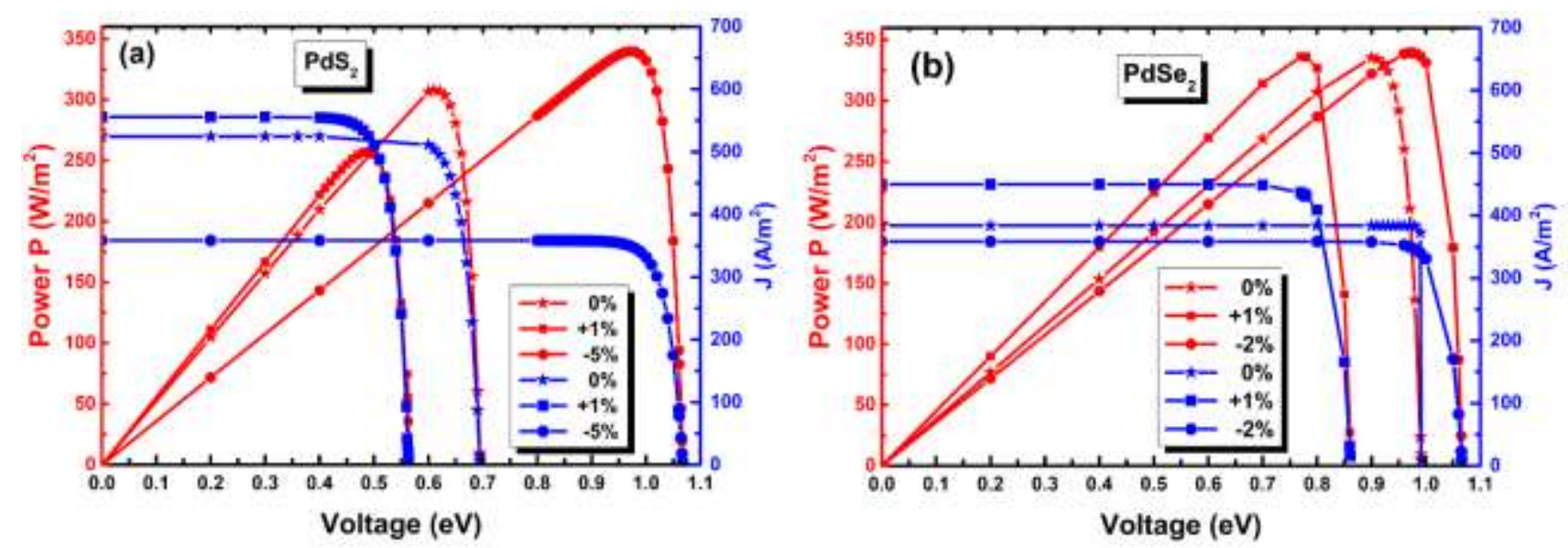
Figure 7. (Colour online) The calculated $J-V$ characteristic and power density curves for pristine and compressive $(-\varepsilon \%)$ or tensile $(+\varepsilon \%))$ loaded strain (maximum efficiency $\eta(\varepsilon \%)$ of strain taken) at $\mathrm{T}=300 \mathrm{~K}$ (a) for penta- $\mathrm{PdS}_{2}$ and (b) for penta-PdSe 2.

As mentioned in the SQ limit theory, the electronic band gap $\left(E_{g}\right)$ plays a pivotal role in the calculation of the material efficiency. Moreover, the maximum possible SQ efficiency limit is specified of $33.70 \%$ in circumstance of semiconductor with $1.34 \mathrm{eV}$ optimum band gap [64]. As seen from the Table 3, the remarkable conversion efficiency for pristine penta-PdS 2 and penta-PdSe 2 is observed and are 30.79 and $33.54 \%$, respectively. Also, the efficiency of penta-PdSe 2 is larger than the efficiency of benchmark GaAs $(\sim 31.4 \%)$ solar cell with single junction. Next, the band gap of both monolayers could be well tuned from $0.34 \mathrm{eV} \sim 1.35 \mathrm{eV}$ under influence of biaxial strain (\%) as shown in Figure $8 \mathbf{( a , ~ b ) . ~ T h e r e f o r e , ~ w e ~ h a v e ~ a l s o ~}$ checked the conversion efficiency under compressive $(-\varepsilon \%)$ and tensile strain $(+\varepsilon \%)$ for both the monolayers and presented in Figure 8 (a, b). It is noted that, when we have exerted compressive strain $(-\varepsilon \%)$ on the penta- $\mathrm{PdQ}_{2}$ the efficiency at certain strain is higher as compared to the pristine (0\%), that of $33.93 \%$ (at $-5 \%$ of penta- $\mathrm{PdS}_{2}$ ) and $-33.94 \%$ (at $-2 \%$ of penta-PdSe 2 ). On other hand, under tensile strain the maximum efficiency arises that of $25.72 \%$ at $+1 \%$ of penta- $\mathrm{PdS}_{2}$ and $33.63 \%$ at $+1 \%$ of penta-PdSe 2 , which is lower than with respective each of pristine $(0 \%)$. This suggests that, by applying biaxial strain on the penta$\mathrm{PdQ}_{2}$ the related SQ efficiency could be tuned. Also, the fabrications of penta-PdQ 2 solar cell have potential to improve photovoltaic performance compared to traditionally use Si-based solar cells.

Table 3. The calculated values of $J_{s c}\left(A / m^{2}\right), P_{\max }\left(W / m^{2}\right)$ and $\eta(\%)$ of penta- $\mathrm{PdQ}_{2}(\mathrm{Q}=\mathrm{S}$, $\mathrm{Se}$ ) for both pristine and loaded strain (\%).

\begin{tabular}{|c|c|c|c|c|}
\hline \multirow{2}{*}{ 2D-System } & Strain (\%) & $\boldsymbol{J}_{\boldsymbol{s c}}\left(\mathbf{A} / \mathbf{m}^{\mathbf{2}}\right)$ & $\boldsymbol{P}_{\boldsymbol{m a x}}\left(\mathbf{W} / \mathbf{m}^{\mathbf{2}}\right)$ & Efficiency $\boldsymbol{\eta} \%$ \\
\hline \multirow{2}{*}{ Penta-PdS2 } & $0 \%$ & 524.67 & 307.96 & $\mathbf{3 0 . 7 9} \%$ \\
\cline { 2 - 5 } & $+1 \%$ & 555.79 & 257.2 & $25.72 \%$ \\
\cline { 2 - 5 } & $-5 \%$ & 358.48 & 339.3 & $33.93 \%$ \\
\hline \multirow{2}{*}{ Penta-PdSe2 } & $0 \%$ & 383.82 & 335.4 & $\mathbf{3 3 . 5 4} \%$ \\
\cline { 2 - 5 } & $+1 \%$ & 449.66 & 336.3 & $33.63 \%$ \\
\cline { 2 - 5 } & $-2 \%$ & 358.48 & 339.48 & $33.94 \%$ \\
\hline
\end{tabular}



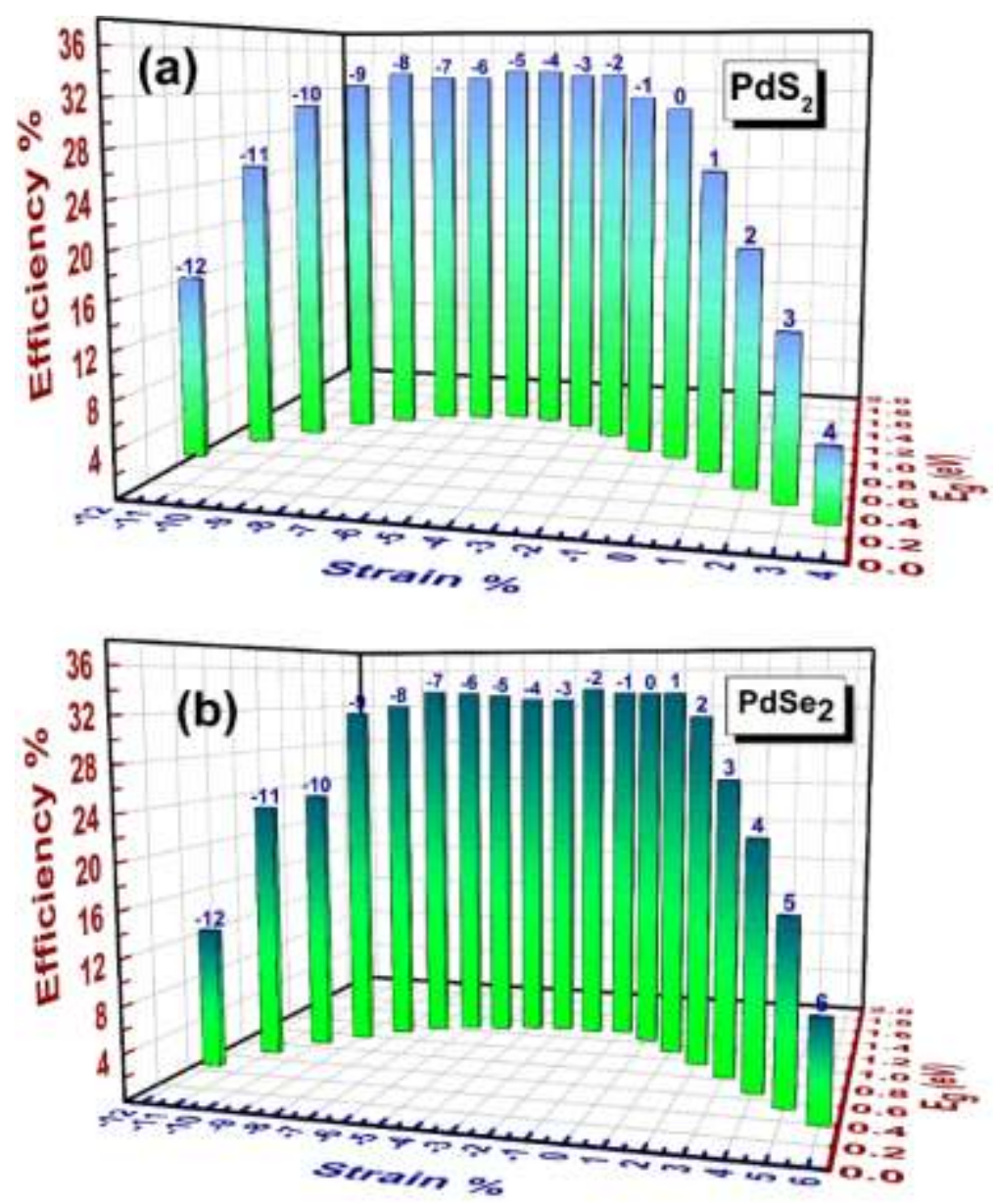

Figure 8. Change in SQ Efficiency $\eta(\%)$ of penta-PdQ 2 monolayers for pristine and loaded strain at $\mathrm{T}=300 \mathrm{~K}$ (a) For penta-PdS 2 and (b) For penta-PdSe 2 .

\subsection{Optical Properties}

In order to evaluate light absorption ability of penta- $\mathrm{PdQ}_{2}$, we have calculated the real $\left(\varepsilon^{\prime}\right)$ and imaginary $(\varepsilon ")$ dielectric functions, absorption coefficient $I(\omega)$, refractive index $n(\omega)$, and reflectivity $R(\omega)$ of penta- $\mathrm{PdQ}_{2}$ monolayers with parallel $(E \| c)$ and perpendicular $(E \perp$ c) electric vector $(\mathrm{E})$. The computation is carried out using the Kramers-Kroning (KK) relationship [65] and results are shown in Figures 9, S8 and S9, ESI $\uparrow$. 

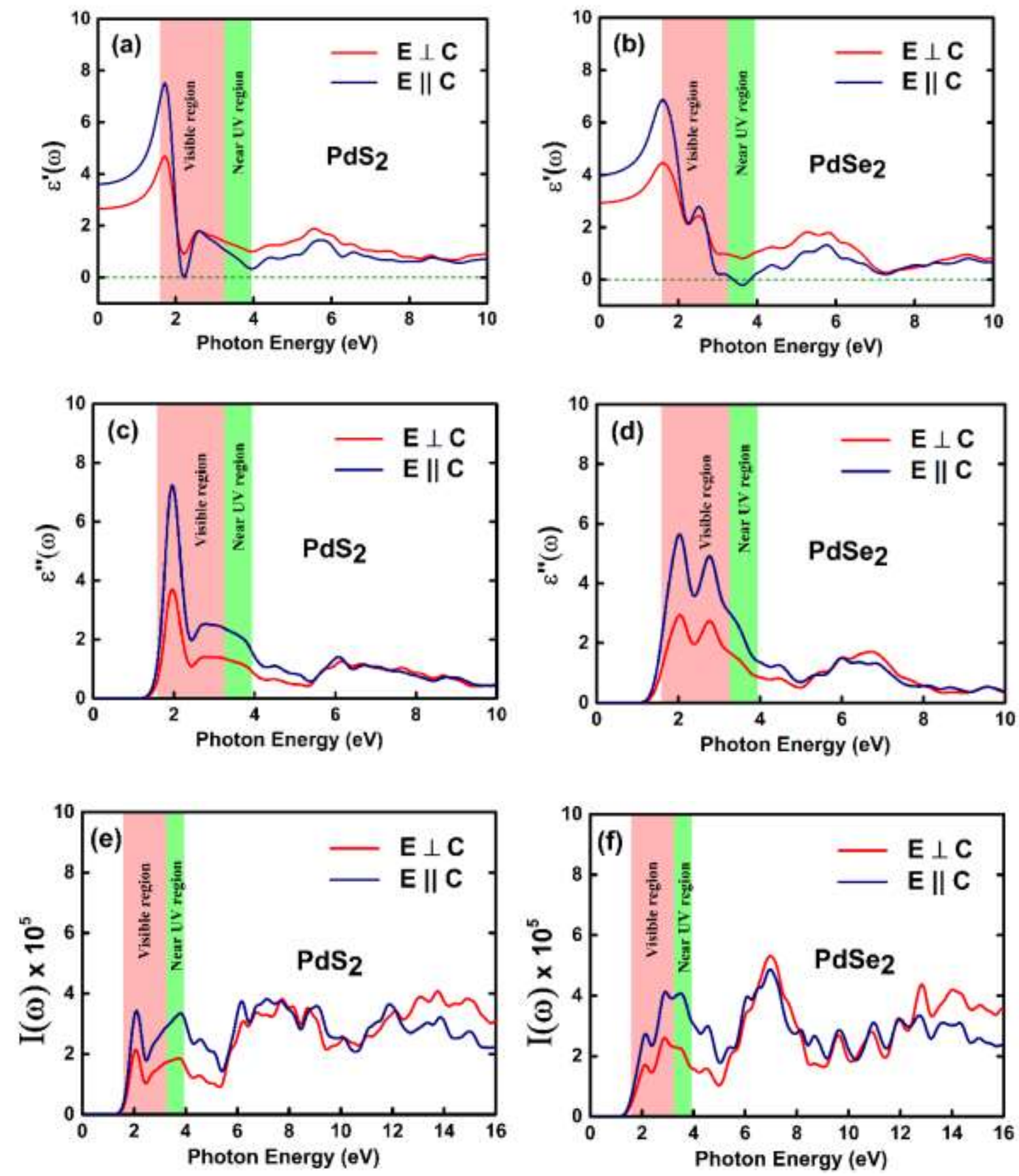

Figure 9. (Colour online) Computed optical properties of penta-PdQ $\mathrm{P}_{2}$ monolayers for parallel and perpendicular polarization $(\mathrm{a}, \mathrm{b})$ real $\left(\varepsilon^{\prime}\right)$ and $(\mathrm{c}, \mathrm{d})$ imaginary $\left(\varepsilon^{\prime \prime}\right)$ parts of complex dielectric function. (e, f) absorption coefficient I $(\omega)$.

In present work, the computed dielectric constants are shown in the large range of photon energy $(\hbar \omega)$ from 0 to $10 \mathrm{eV}$ and absorption coefficients $\mathrm{I}(\omega)$ in 0 to $16 \mathrm{eV}$. The visible $(V I S ; 1.59-3.26 \mathrm{eV})$ and near $U V(U V-A ; 3.26-3.94 \mathrm{eV})$ spectrum regions are 
shaded by the light-pink and light-green colour, respectively. Figure 9 (a, b) shows the graphical image of real $\left(\varepsilon^{\prime}\right)$ and imaginary $\left(\varepsilon^{\prime \prime}\right)$ components of penta-PdQ 2 , that simply gives the idea about the electronic polarizability of the material from the Clausis - Mossotti [66] relation. It can be seen from the Figure $9(\mathbf{a}, \mathbf{b})$, the static dielectric constant $\left(\varepsilon_{\|}(0), \varepsilon_{\perp}(0)\right)$ of penta- $\mathrm{PdS}_{2}$ is $(3.56,2.62)$, while static dielectric constant of penta-PdSe 2 is $4.0\left(\varepsilon_{\|}(0)\right)$ and $2.94\left(\varepsilon_{\perp}(0)\right)$. This means that its $\varepsilon(0)$ of parallel optical vector have more dominant polarizability compared to the perpendicular of $\varepsilon(0)$. The optical peculiarity is strongly connected to the imaginary part of the dielectric function $\varepsilon^{\prime \prime}(\omega)$, which reflects the transition between the energy bands near the $E_{F}$ levels and that would be govern the linear response of the material to light under small wave vector as depicted in Figure 9 (c, d). Therefore, the imaginary part $\varepsilon "(\omega)$ in case of penta-PdS 2 , the one major peak occurred at $1.98 \mathrm{eV}$ in the visible (VIS) region for both $\varepsilon_{\perp}^{\prime \prime}(\omega)$ and $\varepsilon_{\|}^{\prime \prime}(\omega)$ case as plotted in Figure 9 (c). While, in case of penta-PdSe 2 , it is clearly seen (Figure 9 (d)) that there are two intense peaks appeared in the visible region (VIS) at $\hbar \omega=2.04 \mathrm{eV}$ and $2.76 \mathrm{eV}$ due to the electronic transition of ' $4 p$ ' states of Se atom and ' $4 d$ ' states of Pd atom between conduction bands (CBs) and valence bands (VBs) of the electronic band structure. Apparently, the peaks tendency of $\varepsilon_{\perp}^{\prime \prime}(\omega)$ and $\varepsilon_{\|}^{\prime \prime}(\omega)$ are similar on the photon energy spectrum for both penta- $\mathrm{PdQ}_{2}$ monolayers.

The optical absorption spectra of the penta-PdQ 2 were recorded by absorption coefficient $I(\omega)$ vs photon energy $(\mathrm{eV})$ as shown in Figure $9(\mathbf{e}, \mathbf{f})$. For penta-PdQ 2 , the first absorption peak orientation is in the $\sim 1.5-5 \mathrm{eV}$ and second broad peak's orientation is cover the $U V$ region up to photon energy of $16 \mathrm{eV}$. For penta- $\mathrm{PdS}_{2}$, the low energy excitonic peak occur around $2.1 \mathrm{eV}$ in infrared region $(I R)$ and that peak rise towards the visible region at $3.78 \mathrm{eV}$ for both the polarization. The maximum absorption coefficient $I(\omega)$ of penta-PdS $\mathrm{S}_{2}$ for parallel and perpendicular polarization is $3.65 \times 10^{5}$ and $4.04 \times 10^{5}$, respectively (See Figure 9 (e)). While, in case of penta-PdSe 2 , the first peak is located at $2.2 \mathrm{eV}$ in the $I R$ region and kick off in visible region at highest absorption coefficient $\mathrm{I}(\omega)$ of $4.12 \times 10^{5}$. However, with perpendicular polarization the highest coefficient found is about $5.28 \times 10^{5}$ in the $U V$ region (See Figure 9 (f)). Thus, the penta- $\mathrm{PdQ}_{2}$ monolayers have shown prominent absorption in $V I S$ and $U V$ region, which suggest a good prospect of penta- $\mathrm{PdQ}_{2}$ monolayers as $U V$ filters and $U V$ photodetectors.

Next, the computed refractive index $n(\omega)$ and reflectance $R(\omega)$ of penta-PdQ 2 have the same evolution curve as plotted in Figure S8 (a, b), $\mathbf{E S I}^{\dagger}$ and S9 (a, b), $\mathbf{E S I}^{\dagger}$. In 
penta-PdS 2 , we observed static refractive index as $\left(n^{\|}(0), n^{\perp}(0)\right)=(1.90,1.63)$ (see Figure S8 (a), ESI $\left.{ }^{\dagger}\right)$. With the parallel polarization, the $n(\omega)$ is rising in the VIS region up to $\hbar \omega=$ $1.78 \mathrm{eV}$, then going down up to energy of $2.32 \mathrm{eV}$. Similarly, in case of perpendicular polarization it has also gone down in the VIS region with 1.23 of $n(\omega)$ by the same spectrum photon energy. Moreover, the reflectivity $R(\omega)$ is also shown in Figure S9 (a), ESI ${ }^{\dagger}$. The static reflectance $\left(R_{\|}(0), R_{\perp}(0)\right)$ of penta- $\mathrm{PdS}_{2}$ in $V I S$ region is about to $(10 \%, 5 \%)$ and the most elevated peak of reflectance is found to be in the VIS region with reflectance of $31 \%$ as seen in Figure S9 (a, b), ESI ${ }^{\dagger}$. While, for penta-PdSe, the static refractive index $\left(n^{\|}(0)\right.$, $\left.n^{\perp}(0)\right)$ is $(2.0,1.71)$ as shown in Figure S8 (b), ESI ${ }^{\dagger}$, which is also in good agreement with previous reported work on penta-PdSe $\mathrm{Pe}_{2}$ monolayer by the Zhao et al [67] group. The first and highest peak of $n(\omega)$ is trapped in the VIS region at energy of $1.68 \mathrm{eV}$, at that energy the $n^{\|}(\omega)$ is 2.65. The intense peak of $n^{\perp}(\omega)$ is also located at $\hbar \omega=1.68 \mathrm{eV}$ in the VIS region with $n^{\perp}(\omega)$ of 1.68 . The static reflectance $\left(R_{\|}(0), R_{\perp}(0)\right)$ of penta-PdSe 2 in VIS region is about to $(11 \%, 7 \%)$ as seen in Figure S9 (b), $\mathbf{E S I}^{\dagger}$. Also, the highest peak of $R_{\perp}(\omega)$ is occurred in the VIS region with the reflectance of $26 \%$, that reflectance is in good agreement with Zhao et al [67] reported results of penta-PdSe 2 monolayer. Overall, the prominent peaks appeared in the $n(\omega)$ and $R(\omega)$ in the VIS region, that means most of incident light energy is reflected and refracted in VIS region and only small amount of incident energy is absorbed by the material.

\section{Conclusions}

In the summary of the work, the first principles method has been employed to study the band structure, bulk modulus, Young's modulus, transport properties such as carrier mobility and $I-V$ curve, and optical properties of penta- $\mathrm{PdQ}_{2}(\mathrm{Q}=\mathrm{S}, \mathrm{Se})$ monolayers. The positive phonon spectrum gives the ground state dynamical stability of both the materials. The obtained electronic band gap is $0.94 \mathrm{eV}$ and $1.26 \mathrm{eV}$ for penta- $\mathrm{PdS}_{2}$ and penta-PdSe 2 , respectively. From mechanical stability point of view, penta-PdS 2 withstands up to $16 \%(18 \%)$ in $x(y)$ directions, while penta- $\mathrm{PdSe}_{2}$ is up to $17 \%(19 \%)$, which undoubtedly implies that each monolayer is flexible up to that strain. Interestingly, the $I-V$ curve shows the NDC effect beyond the bias voltage of $2.5 \mathrm{~V}$ (for $\mathrm{PdS}_{2}$ ) and $3.0 \mathrm{~V}$ (for $\mathrm{PdSe}_{2}$ ), hence this feature leads us to conclude that penta- $\mathrm{PdQ}_{2}$ monolayers will be consider in future as promising material for NDC-based nanodevices. More importantly, the SQ efficiencies for pristine $\mathrm{PdS}_{2}$ and $\mathrm{PdSe}_{2}$ were $30.79 \%$ and $33.54 \%$, respectively, that could be essential utilized in the solar cell application. The computed optical properties reveal that the absorption range of penta-PdQ 2 
is very broad in $U V$ regions. The maximum absorption coefficient $I(\omega)$ of penta-PdS $\mathrm{S}_{2}$ and penta- $\mathrm{PdSe}_{2}$ are found in the $U V$ region are $4.04 \times 10^{5}$ and $5.28 \times 10^{5}$, respectively. Collectively, due to unique electronic, mechanical, transport and optical properties of penta$\mathrm{PdQ}_{2}$ monolayers, the $2 \mathrm{D}$ materials have application prospect in the arena of semiconducting nanodevices.

\section{Conflicts of interest}

The authors declare that they have no competing interests.

\section{Acknowledgement}

The computer facility developed under DST-FIST Level-I (No.SR/ FST/PSI-097/2006 dated 20th December 2006 and No.SR/FST/PSI-198/2014 dated 21 November 2014) programmes of Department of Science and Technology, Government of India, New Delhi, India and support under DRS-SAP-I (No. F-530/10/DRS/2010 (SAP-I) dated November 2010 and No.F.530/17/DRS-II/2018 (SAP-I), dated 17th April 2018) of University Grants Commission, New Delhi, India are highly acknowledged. DR is thankful for the project fellowship under DRS-II-SAP (No.F.530/17/DRS-II/2018 (SAP-I), dated 17/04/2018) of University Grants Commission, New Delhi, India. S.K.G. would like to thank Science and Engineering Research Board (SERB), India for the financial support (Grant no.: YSS/2015/001269). R.A. thanks the Swedish Research Council (VR-2016-06014 \& VR2020-04410) for financial support.

\section{Author Contributions}

DR, S.K.G and P.N.G studied conception and designed the final approval of the version to be published. DR was associated with the DFT calculations. S.K.G and P.N.G helped to improve the scientific content of the article. All author reviewed the manuscript.

\section{Competing Interests}

The authors declare no competing interest.

\section{References}

[1] Novoselov, KS \& Geim, AK. The rise of graphene. Nat. Mater. 6(3), 183-91 (2007).

[2] Neto, A.C., Guinea, F., Peres, N.M., Novoselov, K.S. \& Geim, A.K. The electronic properties of graphene. Rev. mod. Phys. 81(1), 109 (2009). 
[3] Zhi, C., Bando, Y., Tang, C., Kuwahara, H. \& Golberg, D. Large-scale fabrication of boron nitride nanosheets and their utilization in polymeric composites with improved thermal and mechanical properties. Adv. Mater.21(28), 2889-93 (2009).

[4] Warner, J.H., Rummeli, M.H., Bachmatiuk, A. \& Buchner, B. Atomic resolution imaging and topography of boron nitride sheets produced by chemical exfoliation. ACS nano. 4(3), 1299-304 (2010).

[5] Miró, P., Audiffred, M. \& Heine, T. An atlas of two-dimensional materials. Chem. Soc. Rev. 43(18), 6537-54 (2014).

[6] Kong, X., Liu, Q., Zhang, C., Peng, Z. \& Chen, Q. Elemental two-dimensional nanosheets beyond graphene. Chem. Soc. Rev. 46(8), 2127-57 (2017).

[7] Rasmussen, F.A. \& Thygesen, K. S. Computational 2D materials database: electronic structure of transition-metal dichalcogenides and oxides. J Phys. Chem. C. 119(23), 13169-83 (2015).

[8] Jakubczyk, T., Nogajewski, K., Molas, M.R., Bartos, M., Langbein, W., Potemski, M. \& Kasprzak, J. Impact of environment on dynamics of exciton complexes in a $\mathrm{WS}_{2}$ monolayer. 2D Mater. 5(3), 031007 (2018).

[9] Jariwala, D., Sangwan, V. K., Lauhon, L. J., Marks, T. J. \& Hersam, M. C. Emerging device applications for semiconducting two-dimensional transition metal dichalcogenides. ACS nano. 8(2), 1102-20 (2014).

[10] Novoselov, K. S., Jiang, D., Schedin, F., Booth, T. J., Khotkevich, V. V., Morozov, S. V. \& Geim, A. K. two- dimensional atomic crystals. Proc. Natl. Acad. Sci. U.S.A. 102(30), 10451-3 (2005).

[11] Eda, G., Yamaguchi, H., Voiry, D., Fujita, T., Chen, M. \& Chhowalla, M. Photoluminescence from chemically exfoliated $\mathrm{MoS}_{2}$. Nano Lett. 11(12), 5111-6 (2011).

[12] Lee, Y. H., Zhang, X.Q., Zhang, W., Chang, M.T., Lin, C.T., Chang, K.D., Yu, Y.C., Wang, J.T., Chang, C.S., Li, L.J. \& Lin, T.W. Synthesis of large-area $\mathrm{MoS}_{2}$ atomic layers with chemical vapour deposition. Adv. Mater. 24(17), 2320-5 (2012).

[13] Li, G., Zhang, Y.Y., Guo, H., Huang, L., Lu, H., Lin, X., Wang, Y.L., Du, S. \& Gao, H.J. Epitaxial growth, and physical properties of 2D materials beyond graphene: from monatomic materials to binary compounds. Chem. Soc. Rev. 47(16), 6073-100 (2018).

[14] Long, M., Wang, Y., Wang, P., Zhou, X., Xia, H., Luo, C., Huang, S., Zhang, G., Yan, H., Fan, Z. \& Wu, X. Palladium diselenide long-wavelength infrared photodetector with high sensitivity and stability. ACS nano. 13(2), 2511-9 (2019). 
[15] Gronvold, F., Haraldsen, H.A. \& Kjekshus, A.R. On the sulfides, selenides and tellurides of platinum. Acta Chem. Scand. 14(9), 1879-93 (1960).

[16] Gronvold, F.T. \& Rost, E.R. The crystal structure of $\mathrm{PdSe}_{2}$ and $\mathrm{PdS}_{2}$. Acta Crystallogr. 10(4), 329-31 (1957).

[17] Wang, Y., Li, Y. \& Chen, Z. Not your familiar two-dimensional transition metal disulfide: Structural and electronic properties of the $\mathrm{PdS}_{2}$ monolayer. J. Mater. Chem. C. 3(37), 9603-8 (2015).

[18] Cheng, P.K., Liu, S., Ahmed, S., Qu, J., Qiao, J., Wen, Q. \& Tsang, Y.H. Ultrafast Yb-Doped Fiber Laser Using Few Layers of $\mathrm{PdS}_{2}$ Saturable Absorber. Nanomaterials. 10(12), 2441 (2020).

[19] Oyedele, A.D., Yang, S., Feng, T., Haglund, A.V., Gu, Y., Puretzky, A.A., Briggs, D., Rouleau, C.M., Chisholm, M.F., Unocic, R.R. \& Mandrus, D. Defect-mediated phase transformation in anisotropic two-dimensional $\mathrm{PdSe}_{2}$ crystals for seamless electrical contacts. J. Am. Chem. Soc. 141(22), 8928-36 (2019).

[20] Yu, J., Kuang, X., Gao, Y., Wang, Y., Chen, K., Ding, Z., Liu, J., Cong, C., He, J., Liu, Z. \& Liu, Y. Direct observation of the linear dichroism transition in twodimensional palladium diselenide. Nano Lett. 20(2), 1172-82 (2020).

[21] Liang, Q., Wang, Q., Zhang, Q., Wei, J., Lim, S.X., Zhu, R., Hu, J., Wei, W., Lee, C., Sow, C. \& Zhang, W. High performance, room temperature, ultra broadband photodetectors based on air stable PdSe 2 . Adv. Mater. 31(24), 1807609 (2019).

[22] Luo, L.B., Wang, D., Xie, C., Hu, J.G., Zhao, X.Y. \& Liang, F.X. PdSe $\mathrm{P}_{2}$ multilayer on germanium nanocones array with light trapping effect for sensitive infrared photodetector and image sensing application. Adv. Funct. Mater. 29(22), 1900849 (2019)

[23] Wu, D., Guo, J., Du, J., Xia, C., Zeng, L., Tian, Y., Shi, Z., Tian, Y., Li, X.J., Tsang, Y.H. \& Jie, J. Highly polarization-sensitive, broadband, self-powered photodetector based on graphene/PdSe $/$ germanium heterojunction. ACS nano. 13(9), 9907-17 (2019).

[24] Oyedele, A.D., Yang, S., Liang, L., Puretzky, A.A., Wang, K., Zhang, J., Yu, P., Pudasaini, P.R., Ghosh, A.W., Liu, Z. \& Rouleau, C.M. PdSe 2 : pentagonal twodimensional layers with high air stability for electronics. J. Am. Chem. Soc. 139(40), 14090-7 (2017).

[25] Giubileo, F., Martucciello, N. \& Di Bartolomeo, A. Focus on graphene and related materials. Nanotechnology. 28(41), 410201 (2017). 
[26] Giubileo, F., Grillo, A., Iemmo, L., Luongo, G., Urban, F., Passacantando, M. \& Di Bartolomeo, A. Environmental effects on transport properties of $\mathrm{PdSe}_{2}$ field effect transistors. Mater. Today: Proc. 20, 50-3 (2020).

[27] Radisavljevic, B., Whitwick, M.B. \& Kis, A. Integrated circuits and logic operations based on single-layer $\mathrm{MoS}_{2}$. ACS nano. 5(12), 9934-8 (2011).

[28] Di Bartolomeo, A., Urban, F., Passacantando, M., McEvoy, N., Peters, L., Iemmo, L., Luongo, G., Romeo, F. \& Giubileo, F. A $\mathrm{WSe}_{2}$ vertical field emission transistor. Nanoscale. 11(4), 1538-48 (2019).

[29] Xu, W., Jiang, J., Ma, H., Zhang, Z., Li, J., Zhao, B., Wu, R., Yang, X., Zhang, H., Li, B. \& Shu,0 W. Vapor phase growth of two-dimensional $\mathrm{PdSe}_{2}$ nanosheets for highphotoresponsivity near-infrared photodetectors. Nano Res. 13, 2091-7 (2020).

[30] Lan, Y.S., Chen, X.R., Hu, C.E., Cheng, Y. \& Chen, Q.F. Penta-PdX $2(X=S, S e, T e)$ monolayers: promising anisotropic thermoelectric materials. J. Mater. Chem. A. 7(18), 11134-42 (2019).

[31] Deng, S., Li, L. \& Zhang, Y. Strain modulated electronic, mechanical, and optical properties of the monolayer $\mathrm{PdS}_{2}, \mathrm{PdSe}_{2}$, and $\mathrm{PtSe}_{2}$ for tunable devices. ACS Appl. Nano Mater. 1(4), 1932-9 (2018).

[32] Yang, H., Li, Y., Yang, Z., Shi, X., Lin, Z., Guo, R., Xu, L., Qu, H. \& Zhang, S. Firstprinciples calculations of the electronic properties of two-dimensional pentagonal structure $\mathrm{XS}_{2}(\mathrm{X}=\mathrm{Ni}, \mathrm{Pd}, \mathrm{Pt})$. Vacuum. 174, 109176 (2020).

[33] Qin, D., Yan, P., Ding, G., Ge, X., Song, H. \& Gao, G. Monolayer PdSe2: A promising two-dimensional thermoelectric material. Sci. Rep. 8(1), 1-8 (2018).

[34] Sun, M., Chou, J.P., Shi, L., Gao, J., Hu, A., Tang, W. \& Zhang G. Few-layer PdSe 2 sheets: promising thermoelectric materials driven by high valley convergence. ACS Omega. 3(6), 5971-9 (2018).

[35] Soler, J.M., Artacho, E., Gale, J.D., García, A., Junquera, J., Ordejón, P. SánchezPortal, D. The SIESTA method for ab initio order-N materials simulation. J. Phys. Condens. Matter. 14(11), 2745 (2002).

[36] Perdew, J.P., Burke, K. \& Ernzerhof, M. Generalized gradient approximation made simple. Phys. Rev. Lett. 2877(18), 3865 (1996).

[37] Monkhorst, H.J. \& Pack, J.D., Special points for Brillouin-zone integrations -a reply. Phys. Rev. B. 16, 1748-9 (1976). 
[38] Gonze, X. \& Lee, C. Dynamical matrices, Born effective charges, dielectric permittivity tensors, and interatomic force constants from density-functional perturbation theory. Phys. Rev. B. 55(16), 10355 (1997).

[39] Bardeen, J. \& Shockley, W. Deformation potentials and mobilities in non-polar crystals. Phys. Rev. 80(1), 72 (1950).

[40] Brandbyge, M., Mozos, J.L., Ordejón, P., Taylor, J. \& Stokbro, K. Density-functional method for nonequilibrium electron transport. Phys. Rev. B. 65(16), 165401 (2002).

[41] Ferrer, J., Lambert, C.J., García-Suárez, V.M., Manrique, D.Z., Visontai, D., Oroszlany, L., Rodríguez-Ferradás, R., Grace, I., Bailey, S.W., Gillemot, K. \& Sadeghi, H. GOLLUM: a next-generation simulation tool for electron, thermal and spin transport. New J. Phys. 16(9), 093029 (2014).

[42] Ambrosch-Draxl, C. \& Sofo, J.O. Linear optical properties of solids within the fullpotential linearized augmented planewave method. Comput. Phys. Commun. 175(1), 1-4 (2006).

[43] Bhuyan, P.D., Singh, D., Kansara, S., Yadav, P., Gupta, S.K., Sonvane, Y., Rout, S.K. \& Sinha, E. Experimental and theoretical analysis of electronic and optical properties of $\mathrm{MgWO}_{4}$. J. Mater. Sci. 52(9), 4934-43 (2017).

[44] Akbari, O., Ansari, R. \& Rouhi, S. Mechanical properties of pristine and Fe, V and Ti doped arsenene: density functional theory calculation. Mater. Res. Express. 5(1), 015025 (2018).

[45] Aghdasi, P., Ansari, R., Yousefi, S. \& Goli, M. Structural and mechanical properties of pristine and adsorbed puckered arsenene nanostructures: A DFT study. Superlattices Microstruct. 139, 106414 (2020).

[46] Aghdasi, P. \& Ansari, R. Structural and mechanical properties of $\mathrm{Sb}$ and $\mathrm{SbX}(\mathrm{X}=\mathrm{H}$, F, Cl and Br) monolayers. Solid State Commun. 311, 113849 (2020).

[47] Xiong, W., Huang, K. \& Yuan, S. The mechanical, electronic and optical properties of two-dimensional transition metal chalcogenides $\mathrm{MX}_{2}$ and $\mathrm{M}_{2} \mathrm{X}_{3}(\mathrm{M}=\mathrm{Ni}, \mathrm{Pd} ; \mathrm{X}=\mathrm{S}$, Se, Te) with hexagonal and orthorhombic structures. J. Mater. Chem. C. 7(43), 13518-25 (2019).

[48] Lee, C., Wei, X., Kysar, J.W. \& Hone, J. Measurement of the elastic properties and intrinsic strength of monolayer graphene. Science. 321(5887), 385-8 (2008).

[49] Çakır, D., Peeters, F.M. \& Sevik, C. Mechanical and thermal properties of h-MX $(\mathrm{M}=\mathrm{Cr}, \mathrm{Mo}, \mathrm{W} ; \mathrm{X}=\mathrm{O}, \mathrm{S}, \mathrm{Se}, \mathrm{Te})$ monolayers: A comparative study. Appl. Phys. Lett. 104(20), 203110 (2014). 
[50] Li, J., Medhekar, N.V. \& Shenoy, V.B. Bonding charge density and ultimate strength of monolayer transition metal dichalcogenides. J. Phys. Chem. C. 117(30), 15842-8 (2013).

[51] Swaroop, R., Ahluwalia, P.K., Tankeshwar, K. \& Kumar, A. Ultra-narrow blue phosphorene nanoribbons for tunable optoelectronics. RSC Adv. 7(5), 2992-3002 (2017).

[52] Raval, D., Babariya, B., Gupta, S.K., Gajjar, P.N., \& Ahuja, R. Ultrahigh carrier mobility and light-harvesting performance of $2 \mathrm{D}$ penta-PdX $\mathrm{X}_{2}$ monolayer. J. Mater. Sci. 56(5), 3846-60 (2021).

[53] Jing, Y., Ma, Y., Li, Y. \& Heine, T. GeP 3 : A small indirect band gap 2D crystal with high carrier mobility and strong interlayer quantum confinement. Nano Lett. 17(3), 1833-8 (2017).

[54] Qiao, J., Kong, X., Hu, Z.X., Yang, F. \& Ji, W. High-mobility transport anisotropy and linear dichroism in few-layer black phosphorus. Nat. Commun. 5(1), 1-7 (2014).

[55] Bruzzone, S. \& Fiori, G. Ab-initio simulations of deformation potentials and electron mobility in chemically modified graphene and two-dimensional hexagonal boronnitride. Appl. Phys. Lett. 99(22), 222108 (2011).

[56] Xu, B. \& Dubi, Y. Negative differential conductance in molecular junctions: an overview of experiment and theory. J. Phys. Condens. Matter. 27(26), 263202 (2015).

[57] Mathews, R.H., Sage, J.P., Sollner, T.G., Calawa, S.D., Chen, C.L., Mahoney, L.J., Maki, P.A. \& Molvar, K.M. A new RTD-FET logic family. Proc. IEEE. 87(4), 596605 (1999).

[58] Shockley, W. The Shockley-Queisser limit. J. Appl. Phys. 32, 510-9 (1961).

[59] Som, N.N., Sampath, P.M., Dabhi, S.D., Mankad, V., Shinde, S., Attygalle, M.L. \& Jha, P.K. Strain and layer modulated electronic and optical properties of low dimensional perovskite methylammonium lead iodide: Implications to solar cells. Sol Energy. 173, 1315-22 (2018).

[60] Som, N.N., Sharma, V., Mankad, V., Attygalle, M.L. \& Jha, P.K. Role of $\mathrm{CuAlO}_{2}$ as an absorber layer for solar energy converter. Sol Energy. 193, 799-805 (2019).

[61] Guo, M., Xie, K., Lin, J., Yong, Z., Yip, C.T., Zhou, L., Wang, Y. \& Huang, H. Design and coupling of multifunctional $\mathrm{TiO}_{2}$ nanotube photonic crystal to nanocrystalline titania layer as semi-transparent photoanode for dye-sensitized solar cell. Energy Environ. Sci. 5(12), 9881-8 (2012). 
[62] Bremner, S.P., Levy, M.Y. \& Honsberg, C.B. Analysis of tandem solar cell efficiencies under AM1. 5G spectrum using a rapid flux calculation method. Prog Photovolt. 16(3), 225-33 (2008).

[63] Yin, W.J., Shi, T. \& Yan, Y. Unusual defect physics in $\mathrm{CH}_{3} \mathrm{NH}_{3} \mathrm{PbI}_{3}$ perovskite solar cell absorber. Appl. Phys. Lett. 104(6), 063903 (2014).

[64] Rühle, S. Tabulated values of the Shockley-Queisser limit for single junction solar cells. Sol Energy. 130, 139-47 (2016).

[65] Veal, B.W., Paulikas, A.P. Optical properties of molybdenum. I. Experiment and Kramers-Kronig analysis. Phys. Rev. B. 10(4), 1280 (1974).

[66] Aspnes, D.E. Optical properties of thin films. Thin Solid Films. 89(3), 249-62 (1982).

[67] Zhao, X., Zhao, Q., Zhao, B., Dai, X., Wei, S. \& Ma, Y. Electronic and optical properties of $\mathrm{PdSe}_{2}$ from monolayer to trilayer. Superlattices Microstruct. 142, 106514 (2020). 


\section{Supplementary Files}

This is a list of supplementary files associated with this preprint. Click to download.

- 30thOCTSupDSP.docx 